ARTICLE

https://doi.org/10.1038/s41467-018-07801-x

\title{
A miR-150/TET3 pathway regulates the generation of mouse and human non-classical monocyte subset
}

Dorothée Selimoglu-Buet (10 1, Julie Rivière ${ }^{1,2}$, Hussein Ghamlouch ${ }^{1,2}$, Laura Bencheikh ${ }^{1,2}$, Catherine Lacout ${ }^{1,2}$, Margot Morabito1,2, M'boyba Diop (10 3, Guillaume Meurice ${ }^{3}$, Marie Breckler ${ }^{3}$, Aurélie Chauveau 1,4, Camille Debord ${ }^{1,5}$, Franck Debeurme ${ }^{1,2}$, Raphael Itzykson ${ }^{1,6}$, Nicolas Chapuis ${ }^{7}$, Christophe Willekens (1) 1,8,9, Orianne Wagner-Ballon ${ }^{10}$, Olivier A. Bernard ${ }^{1,2}$, Nathalie Droin (1) ${ }^{1,2,3}$ \& Eric Solary ${ }^{1,2,9}$

Non-classical monocyte subsets may derive from classical monocyte differentiation and the proportion of each subset is tightly controlled. Deregulation of this repartition is observed in diverse human diseases, including chronic myelomonocytic leukemia (CMML) in which nonclassical monocyte numbers are significantly decreased relative to healthy controls. Here, we identify a down-regulation of hsa-miR-150 through methylation of a lineage-specific promoter in CMML monocytes. Mir150 knock-out mice demonstrate a cell-autonomous defect in nonclassical monocytes. Our pulldown experiments point to Ten-Eleven-Translocation-3 (TET3) mRNA as a hsa-miR-150 target in classical human monocytes. We show that Tet3 knockout mice generate an increased number of non-classical monocytes. Our results identify the miR150/TET3 axis as being involved in the generation of non-classical monocytes.

\footnotetext{
${ }^{1}$ INSERM U1170, Gustave Roussy Cancer Center, 94805 Villejuif, France. ${ }^{2}$ Université Paris-Sud, Faculté de Médecine, 94270 Le Kremlin-Bicêtre, France. ${ }^{3}$ INSERM US23, CNRS UMS 3655, Gustave Roussy Cancer Center, 94805 Villejuif, France. ${ }^{4}$ Laboratoire d'Hématologie, Centre Hospitalier Régional Universitaire, 29200 Brest, France. ${ }^{5}$ Laboratoire d'Hématologie, Centre Hospitalier Régional Universitaire, 44000 Nantes, France. ${ }^{6}$ Département d'Hématologie, Hôpital Saint-Louis, Assistance Publique-Hôpitaux de Paris, 75010 Paris, France. ${ }^{7}$ Département d'Immuno-Hématologie, Institut Cochin, 75014 Paris, France. ${ }^{8}$ Université Paris Diderot, 75004 Paris, France. ${ }^{9}$ Département d'Hématologie, Gustave Roussy Cancer Center, 94805 Villejuif, France. 10 Département d'Hématologie et d'Immunologie Biologiques, Hôpital Henri-Mondor, 94010 Créteil, France. These authors contributed equally: Nathalie Droin, Eric Solary. Correspondence and requests for materials should be addressed to D.S.-B. (email: dorothee.selimoglubuet@gustaveroussy.fr) or to E.S. (email: eric.solary@gustaveroussy.fr)
} 
P eripheral blood monocytes are components of the mononuclear phagocyte system that is involved in rapid recognition and clearance of invading pathogens and in tissue integrity maintenance. In mice and humans, the peripheral blood monocyte pool is phenotypically and functionally heterogeneous. Using Ly6C surface antigen, two main monocyte subsets are depicted in mice ${ }^{1}$ Ly6Chigh, CX3CR $1^{\text {low }}$, CCR2 $2^{\text {high }}, \mathrm{CD} 43^{\text {low }}$, $\mathrm{CD} 2 \mathrm{~L}^{+}$classical monocytes are progenitors of inflammatory and some tissue-resident macrophages. Ly6Clow, CX3CR1 $1^{\text {high }}$, CCR2 ${ }^{\text {low }}, \mathrm{CD} 43^{\text {high }}, \mathrm{CD} 62 \mathrm{~L}$ nonclassical monocytes patrol the resting endothelium to maintain vascular homeostasis ${ }^{2}$, protect against the seeding of metastatic tumor cells, and populate tissues to contribute to wound healing ${ }^{3}$. Based on cell surface expression of CD14 and CD16, human circulating monocytes are divided into three subsets ${ }^{4}$. Classical $\mathrm{CD} 14^{+}, \mathrm{CD} 16^{-}$monocytes, which represent roughly $85 \%$ of total human monocytes, are analogous to Ly6Chigh mouse monocytes, express high level of the chemokine receptor CCR2, show a strong inflammatory response to lipopolysaccharides, and are rapidly recruited to inflamed and infected tissues. They are distinguished from $\mathrm{CD}_{14}{ }^{+}, \mathrm{CD}_{16}{ }^{+}$ intermediate monocytes and $\mathrm{CD} 14^{\text {low }}, \mathrm{CD}_{1}{ }^{+}$nonclassical monocytes, which are more probably analogous to Ly6Clow monocytes, express higher levels of CX3CR1 receptor, and remain longer in the blood to patrol on the luminal part of the endothelium wall ${ }^{2,5}$.

Monocyte subsets arise from a multipotent hematopoietic stem cell via a series of progenitors with increasingly restricted lineage potential ${ }^{6}$. Detailed steps of this differentiation pathway remain unclear as recent studies have challenged traditional hierarchical models by suggesting that some progenitors could make lineage commitment earlier than initially thought ${ }^{7-9}$. Accordingly, two independent pathways of monocyte generation were recently depicted in mice, leading to functionally distinct Ly6Chigh monocytes whose production varies in steady state and stress condition ${ }^{10,11}$. The origin of circulating monocyte subsets has long been controversial until a consensus model emerged supporting a hierarchical relationship in which most if not all Ly6C low monocytes derive from Ly6Chigh monocytes and the transcription factor $\mathrm{Nr} 4 \mathrm{a} 1$ is the master regulator of the differentiation and survival of Ly6C $C^{\text {low }}$ monocyte subset ${ }^{12,13}$. Gene expression patterns depicted in human monocyte subsets also suggest a common origin 14,15 . Finally, in vivo deuterium labeling supports a model of sequential transition from classical to intermediate and nonclassical monocytes, at steady state and in response to endotoxemia ${ }^{16}$. However, the molecular mechanisms that drive the generation of nonclassical monocytes in humans are only partly understood.

Some pathological conditions offer opportunities to explore human monocyte biology and more specifically the relationships between human monocyte subsets. A depletion of the slanpositive nonclassical monocytes is observed in patients with hereditary diffuse leukoencephalopathy with axonal spheroids, a rare neurologic disease associated with inactivating mutations in the colony-stimulating factor-1 receptor (CSF1R) tyrosine kinase domain ${ }^{17}$. Conversely, infectious and inflammatory diseases promote an increase in the fraction of intermediate and nonclassical monocytes ${ }^{17-19}$. We detected an abnormal repartition of monocyte subsets in the blood of patients with a chronic myeloid malignancy, especially those with a chronic myelomonocytic leukemia (CMML) ${ }^{20}$. CMML is a clonal hematopoietic stem cell malignancy related to the accumulation of recurrent somatic mutations in epigenetic regulators, splicing genes, and signaling genes, mostly of the RAS pathway ${ }^{21-24}$. Flow cytometry analysis of peripheral blood monocytes shows a strong decrease in intermediate and nonclassical monocyte subsets, whatever the genetic alterations, which rapidly distinguishes a CMML from a reactive monocytosis $20,25,26$. Altered DNA methylation is another characteristic feature of CMML cells that contributes to disease phenotype ${ }^{27}$. Hypomethylating agents, which are commonly used to treat these patients ${ }^{28}$, can restore a balanced hematopoiesis by demethylating leukemic cell DNA without having any impact on variant allele frequencies in the leukemic population ${ }^{24}$.

By profiling microRNA (miRNA) expression in peripheral blood monocytes of CMML patients, we identify the downregulation of hsa-miR-150 expression as a characteristic feature of CMML monocytes. Analysis of Mir150 knockout mice detects a cell-autonomous abnormal repartition of monocyte subsets. We show that hsa-miR-150 down-regulation is related to the hypermethylation of a myeloid lineage-specific promoter in MIR150 gene in CMML classical monocytes. In mice and in humans, this down-regulation prevents the generation of intermediate and nonclassical monocytes through up-regulating the ten-eleventranslocation-3 (TET3) protein in classical monocytes. These results highlight a mechanism of monocyte subset differentiation that operates in both mice and humans.

\section{Results}

hsa-miR-150 expression is down-regulated in CMML monocytes. To elucidate the potential role of miRNAs in the pathogenesis of CMML, we examined the expression of 851 human miRNAs in sorted peripheral blood monocytes $\left(\mathrm{CD} 14^{+}\right)$collected from a learning cohort of 33 severe CMML patients (Supplementary Table 1) and 5 healthy donors using microarrays. Unsupervised clustering of miRNA profiles separated CMML from control samples (Supplementary Figure 1A). Using an absolute 2-fold change and a $P$ value $<0.05$ as thresholds, we observed a downregulation of hsa-miR-150 and hsa-miR-451 while hsa-miR-494 appeared to be up-regulated in CMML compared to control samples (Fig. 1a, b and Supplementary Figure 1B). Quantitative PCR (qPCR) validation using either RNU-6B miRNA (Fig. 1c) or RPL32 or PPIA housekeeping genes (Supplementary Figure 1C) as normalizers confirmed the down-regulation of hsa-miR-150 and hsa-miR-451 in these samples, but not the up-regulation of hsa-miR-494. To further validate these results, we measured the expression of hsa-miR-150 and hsa-miR-451 in sorted peripheral blood $\mathrm{CD} 14^{+}$of an independent cohort of $139 \mathrm{CMML}$ patients at diagnosis (Supplementary Table 1), using CD14+ sorted from 24 healthy donor blood samples as controls. While the downregulation of hsa-miR-150 was confirmed in this cohort, we did not validate the down-regulation of hsa-miR-451 nor the upregulation of hsa-miR-494 (Fig. 1d and Supplementary Figure 1D). Therefore, we focused our subsequent efforts in understanding the mechanisms and consequences of hsa-miR150 down-regulation in CMML monocytes. Importantly, the expression level of hsa-miR-150 was not associated with specific disease features, including age at diagnosis, white blood cell count, absolute number of monocytes or neutrophils, hemoglobin level, platelet count (Supplementary Figure 1E), bone marrow blast cell infiltration that distinguishes CMML-0 from CMML-1 and CMML-2 (Supplementary Figure $1 \mathrm{~F})^{29}$, and the presence of somatic mutations in TET2, SRSF2, ASXL1, RUNX1 and genes of the RAS family (NRAS and KRAS) (Supplementary Figure 1G). Together, these results indicated that hsa-miR-150 was downregulated in peripheral blood monocytes of CMML patients, regardless of disease clinical and biological features.

Mir150-I- mice show an abnormal monocyte subset repartition. Analysis of germline Mir $150^{-1-}$ mice identified a role for mmumiR-150 in B lymphopoiesis ${ }^{30}, \mathrm{CD}^{+} \mathrm{T}$ cell fate and function ${ }^{31}$, natural killer (NK) cell differentiation ${ }^{32,33}$, and megakaryocytic commitment ${ }^{34}$. Nevertheless, these animals demonstrate largely 

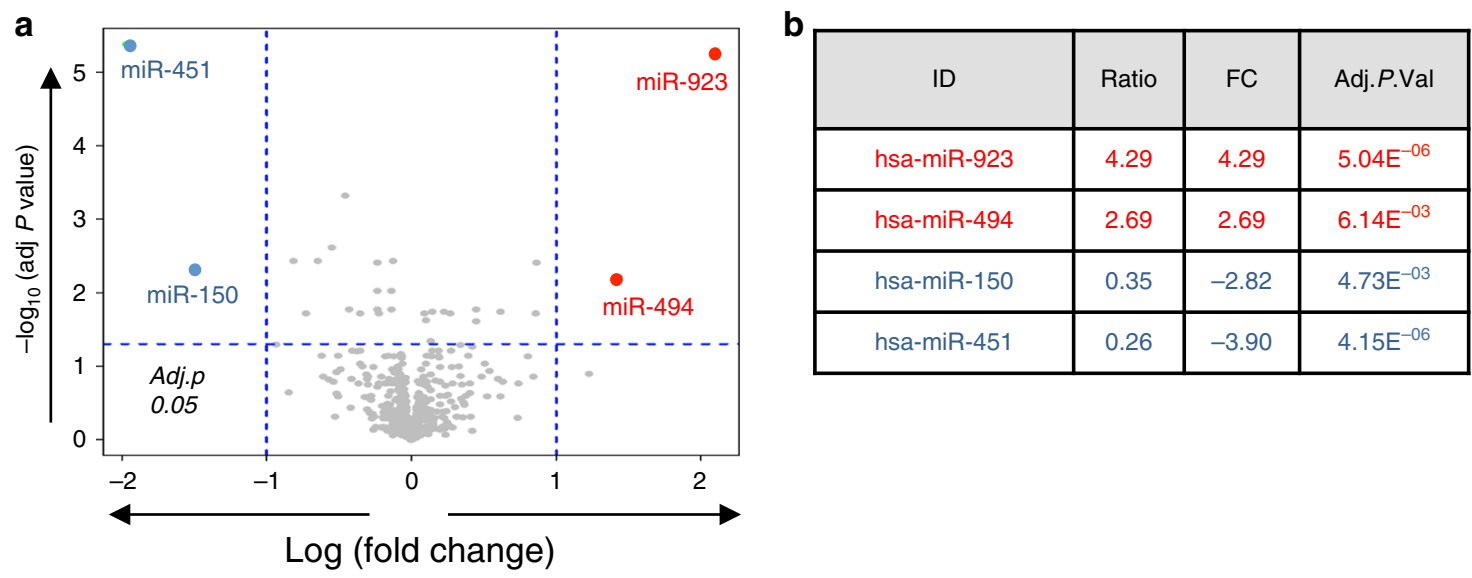

c
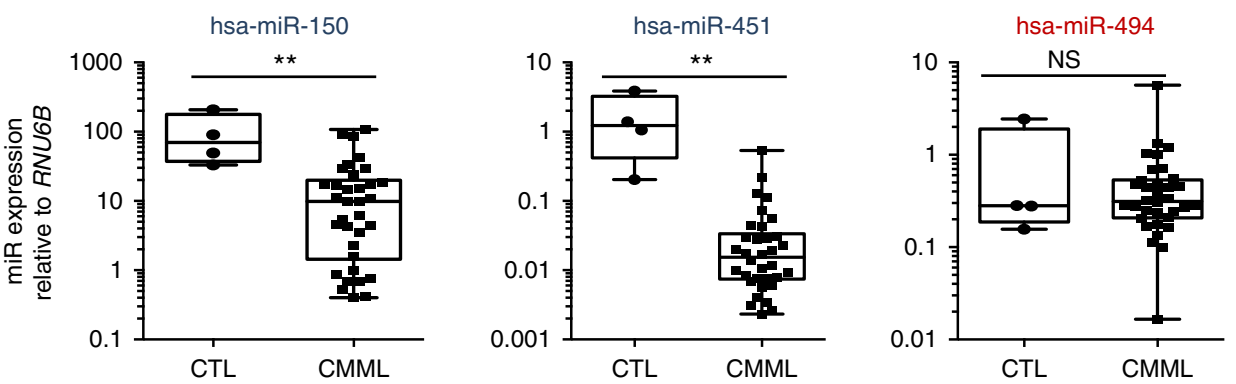

d
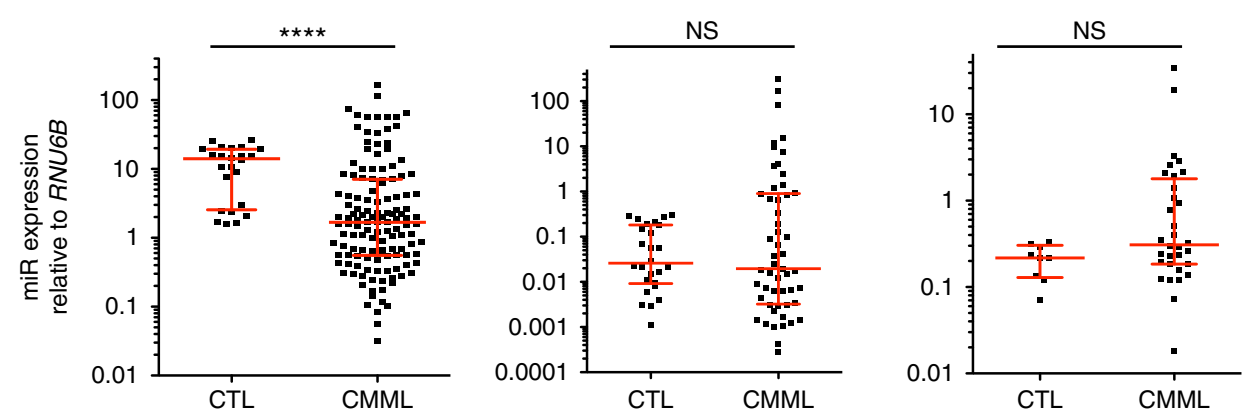

Fig. 1 Differentially expressed miRNA from control and CMML patient-sorted CD14+. a Volcano plot showing the differentially expressed miRNAs in CD14+ cell-sorted monocytes from CMML $(n=33)$ and healthy donor $(n=5+$ pool of five samples) peripheral blood samples (learning cohort), as measured using v12.0 Agilent microarray. $X$-axis, $\log 2$ fold-change value; $Y$-axis, $-\log 10$ (adjusted $P$ value). $\mathbf{b}$ List of the four miRNAs identified as deregulated in the learning cohort. Of note, hsa-miR-923 up-regulation is a known artifact of the v12.0 version of these microarrays. $\mathbf{c}$ Box plot showing the relative expression of hsa-miR-150, hsa-miR-451, and hsa-miR-494 analyzed by qRT-PCR in samples of the learning cohort (center line: median; whiskers: min to max). $\mathbf{d}$ qRTPCR analysis of the relative expression levels of hsa-miR-150 (CTL $=24 ; C M M L=133)$, hsa-miR-451 $(C T L=24, C M M L=53)$ and hsa-miR-494 $(C T L=9$; $\mathrm{CMML}=32)$ in an independent validation cohort. Red lines, median with interquartile range; Mann-Whitney test: ${ }^{\star \star} P<0.01$; ${ }^{\star \star \star \star} P<0.0001$. NS nonsignificant

normal steady-state hematopoiesis and peripheral blood cell counts $^{35}$. We used these mice (Supplementary Figure 2A) to determine if mmu-miR-150 deletion could affect the monocytic lineage with age, as aging is an important component of CMML pathophysiology ${ }^{24}$. In Mir $150^{-1-}$ animals kept aging for up to 2 years, we detected previously described changes in B cell differentiation in the spleen and a slight increase in peripheral blood leukocytes count with age (Supplementary Figure 2B), without any significant change in peripheral blood (Supplementary Figure 2C) and bone marrow (Supplementary Figure 2D) monocyte counts, nor in peripheral blood neutrophil and lymphocyte counts (Supplementary Figure 2E), nor in spleen weight. Thus, Mir150 deletion did not generate a CMML-like phenotype in mice. Mouse monocytes were further analyzed as side scatter ${ }^{\text {low, }}$ $\mathrm{CD}_{4} 5^{+}, \mathrm{B}_{220^{-}}, \mathrm{CD}^{-}, \mathrm{NKP}^{-} 6^{-}, \mathrm{Ly}_{6 \mathrm{G}^{-}}, \mathrm{CD} 115^{+}$, and $\mathrm{CD} 11 \mathrm{~b}^{+}$ cells and subdivided into Ly6Chigh, $\mathrm{CD} 43^{\text {low }}$ classical and Ly6Clow, $\mathrm{CD}^{2} 3^{+}$nonclassical monocytes (Supplementary Figure $2 \mathrm{~F}$ ). We tested two multiparametric flow cytometry strategies, either an exclusion gating strategy (Supplementary Figure 3A) or a CD115 expression-based analysis (Supplementary Figure 3B), to quantify monocyte subsets in mouse peripheral blood and bone marrow. Both methods revealed an abnormal repartition of monocyte subsets in Mir $150^{-1-}$ mice (Supplementary Figure 3C). More specifically, we observed a significant decrease in the fraction and absolute number of Ly6C ${ }^{\text {low }}$ cells in the bone marrow (Fig. 2a-c) and in the peripheral blood (Fig. 2d-f) of younger ( $<6$ months) as well as older ( $>6$ months) animals. This abnormal repartition of monocyte subsets was not related to an increased apoptosis of Ly6Clow compared to Ly6Chigh cells, as determined by Annexin V and 7-amino-actinomycin D staining of blood and 

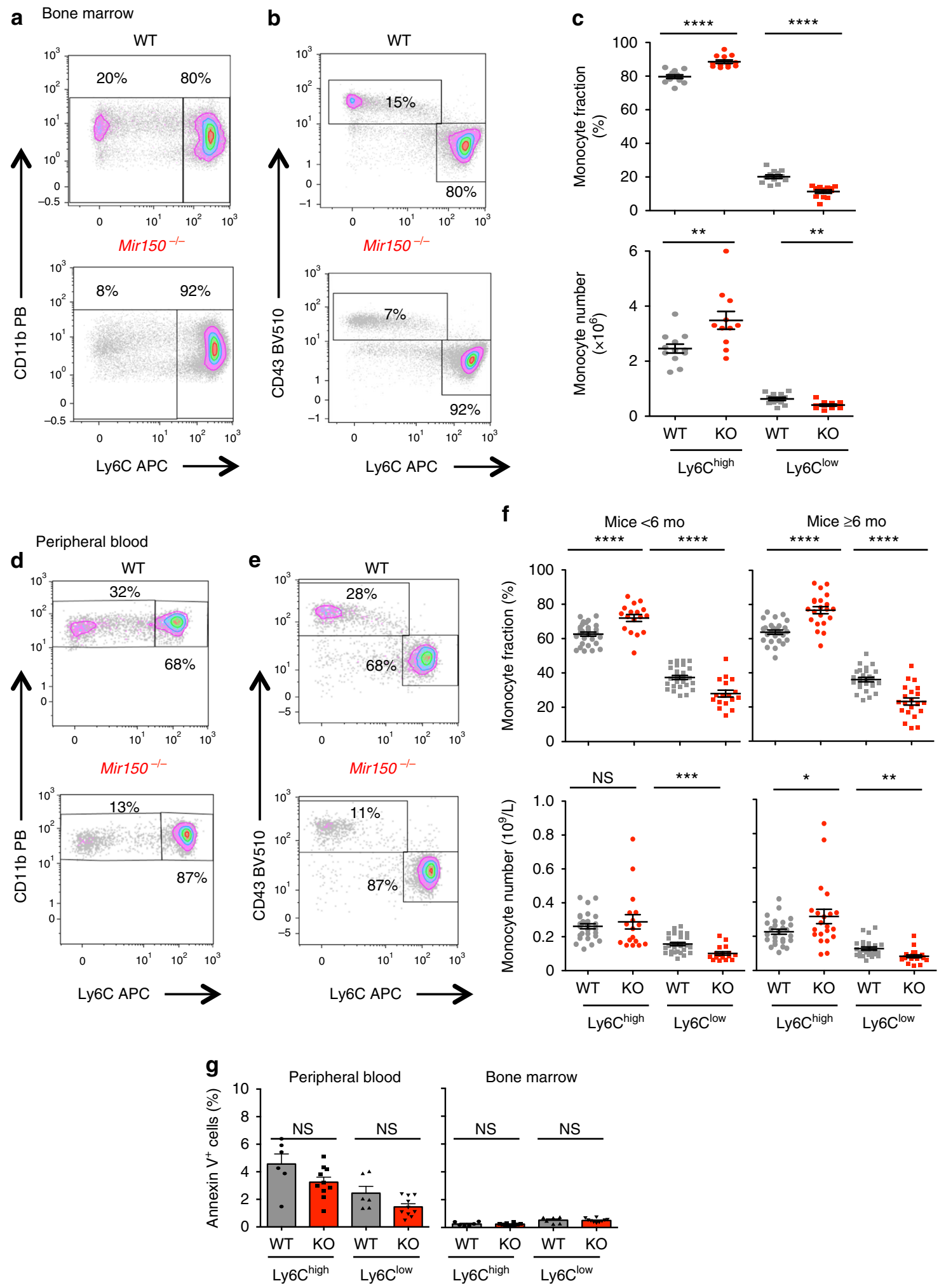

bone marrow monocytes (Fig. $2 \mathrm{~g}$ and Supplementary Figure 4A). Also, this abnormal repartition of monocyte subsets appeared in the absence of any change in the fraction of common myeloid progenitor, granulocyte-monocyte progenitor, and megakaryocyticerythroid progenitor (MEP) cells as well as $\mathrm{LSK}\left(\mathrm{Lin}^{-}, \mathrm{Sca}^{+}, \mathrm{Kit}^{+}\right)$ and LK $\left(\mathrm{Lin}^{-}, \mathrm{Kit}^{+}\right.$) progenitor cells in the bone marrow (Supplementary Figure 4B, C). Finally, we did not detect any significant change in the mean fluorescence intensity of CD115 and CX3CR1 at the surface of Ly6C low Mir150-1- mouse monocytes (Supplementary Figure 4D).

Cell-autonomous effect of mmu-miR-150 on subset generation. To further explore the role of mmu-miR-150 in mouse monocyte subset generation, we transferred bone marrow cells of wild-type and Mir $150^{-1-}$ animals into lethally irradiated wild-type and Mir $150^{-1-}$-irradiated recipients. Six weeks after transplantation, the 
Fig. 2 Abnormal repartition of monocyte subsets in Mir150-/- (KO) mice. a, b Representative flow cytometry scatter plot of CD11b ${ }^{+}$monocytes (a) or CD43 ${ }^{+}$monocytes (b) gated on Ly6C expression in bone marrow of a wild-type (WT) and a Mir $750^{-/-}$mouse. c Flow cytometry quantification of Ly6Chigh and Ly6Clow monocyte subsets in the bone marrow of WT and Mir150-/- (KO) animals (12/group) expressed as a fraction of total monocytes (upper panels) or absolute number per mouse femur (lower panels). Mean \pm SEM, two-tailed unpaired $t$ test: ${ }^{\star \star} P<0.01$; ${ }^{\star \star \star \star} P<0.0001$. d, e. Representative flow cytometry scatter plot of $\mathrm{CD}_{11 b^{+}}$monocytes (d) or CD43+ monocytes (e) gated on Ly6C expression in the peripheral blood of a WT and Mir150-/mouse. $\mathbf{f}$ Flow cytometry quantification of Ly $6 \mathrm{C}^{\text {high }}$ and Ly $6 \mathrm{C}^{\mathrm{low}}$ monocyte subsets in the peripheral blood of young $(<6 \mathrm{months}, \mathrm{WT}=28, \mathrm{KO}=17)$ or older (6-26 months, WT =26; KO=21 KO) animals expressed as fraction of total monocytes (upper panels) or absolute number per mouse (lower panels). Mean $\pm \mathrm{SEM}$, two-tailed unpaired $t$ test: ${ }^{\star} P<0.05 ;{ }^{\star \star} P<0.01$; ${ }^{\star \star \star} P<0.001 ;{ }^{\star \star \star \star} P<0.0001$. $\mathrm{g}$ Flow cytometry measurement of the fraction of Annexin $\mathrm{V}^{+}$cells among Ly6Chigh and Ly6Clow monocytes in the peripheral blood and bone marrow of 6 WT (gray bars) and 10 Mir150-/- (red bars) animals (mean \pm SEM). NS nonsignificant

repartition of monocyte subsets was normal in the blood of wildtype and Mir $150^{-1-}$ mice engrafted with wild-type bone marrow cells, whereas the fraction of peripheral blood Ly6 $\mathrm{C}^{\text {low }}$ monocytes was decreased in the blood of wild-type and Mir150-/- mice engrafted with Mir150-1- bone marrow cells (Fig. 3a, b). We then transferred various ratios $(50 / 50,90 / 10,10 / 90)$ of bone marrow cells collected from CD45.1 wild-type and CD45.2 Mir150-/- mice, respectively, into lethally irradiated CD45.1 recipients (Fig. 3c). Eight weeks later, the fraction of $\mathrm{CD} 45.2^{+}$ cells (among total CD45 ${ }^{+}$cells) was roughly identical to those defined in the transplanted cell mixture (Fig. 3d). The CD45.1/ CD45.2 ratio in lymphocyte and monocyte populations recovered at 8 weeks post-transplantation was also similar to that defined prior to injection (Fig. 3e). Similar observations were made 12 and 40 weeks after transplantation (Supplementary Figure $5 \mathrm{~A}-\mathrm{D})$. Focusing on monocyte subsets, Ly6 $6 \mathrm{C}^{\text {high }}$ cell fraction was higher among engrafted 45.2 Mir150-1- monocytes than among engrafted CD45.1 wild-type cells, and Ly6Clow cell fraction showed the opposite repartition (Fig. 3f). These experiments suggested that the abnormal repartition of monocyte subsets observed in Mir150-1- mice was a cell-autonomous phenotype, with Mir150 gene deletion partially preventing the generation of

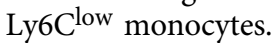

To further verify that the decrease in Ly6Clow subset was related to Mir150 loss, we conducted an in vivo rescue of mmumiR-150 expression in the hematopoietic tissue. Lineage-negative $\left(\mathrm{Lin}^{-}\right)$bone marrow cells from Mir150-1--deficient mice were transduced with a retroviral vector encoding mmu-miR-150 and green fluorescent protein (GFP), sorted to validate mmu-miR-150 overexpression compared to WT $\mathrm{Lin}^{-}$cells, and injected to lethally irradiated wild-type recipients $(n=16)$ without sorting $\mathrm{GFP}^{+}$cells (about $25 \%$ of $\mathrm{Lin}^{-}$cells) (Fig. 3g). A variable fraction of $\mathrm{GFP}^{+}$cells, ranging from 10 to $70 \%$ of total cells, was detected in the blood of six engrafted animals, showing the restoration of a normal monocyte subset repartition in $\mathrm{GFP}^{+}$, but not $\mathrm{GFP}^{-}$, monocytes (Fig. 3h).

Since the phenotype of Mir $150^{-1-}$ mice is not the complete reduction of nonclassical monocytes otherwise observed in $N r 4 a 1^{-1-12,13}$ or $C e b p b^{-1-36,37}$ mice, we explored the conversion ability of Ly6 $\mathrm{C}^{\text {high }}$ by transfer experiment. A 1:1 ratio of wild-type CD45.1 and Mir150-1- CD45.2 Ly6Chigh monocytes were injected into transgenic mice that express GFP under the direction of the human ubiquitin $\mathrm{C}$ promoter ${ }^{38}$. After $40 \mathrm{~h}$, we analyzed monocyte subsets by flow cytometry in the peripheral blood, the spleen, and the bone marrow. Dead cells and cells expressing Ly6G, CD3, B220, and NK1.1 were excluded. Ly6C high and Ly6 $\mathrm{C}^{\text {low }}$ subsets were quantified in CD45.1 ${ }^{+}$and $\mathrm{CD} 45.2^{+}, \mathrm{GFP}^{-}, \mathrm{CD}_{115^{+}}, \mathrm{CD}_{11 \mathrm{~b}}{ }^{+}$monocyte populations. A defective conversion of Ly6 $\mathrm{C}^{\text {high }}$ into Ly6Clow subset was observed in every studied tissue among Mir150-l- CD45.2 compared to wild-type CD45.1 monocytes (Fig. 3i, $j$ and Supplementary Figure 5). Of note, the 1:1 ratio of wild-type and Mir150- ${ }^{-}$monocytes at injection was conserved at $40 \mathrm{~h}$, indicating that Mir150 gene deletion did not promote apoptosis in this time frame.

miR-150 is differentially expressed in monocyte subsets. Analysis of monocyte subsets sorted from wild-type mouse peripheral blood showed a 2-fold higher expression of mmu-miR-150 in Ly6C ${ }^{\text {low }}$ compared to Ly6 $\mathrm{C}^{\text {high }}$ monocytes (Fig. 4a and Supplementary Figure 6A). In humans, compared to sorted classical monocytes, hsa-miR-150 expression was 1.6-fold higher in intermediate monocytes and more than 10-fold higher in nonclassical monocytes, respectively (Fig. $4 \mathrm{~b}$ and Supplementary Figure 6B). We transduced human $\mathrm{CD} 34^{+}$cells with a lentiviral vector encoding GFP and either hsa-miR-150 (miR-150), or a scrambled shRNA (sh-scr) or a shRNA targeting hsa-miR-150 (sh-miR-150). We cultured these cells in a liquid medium with stem cell factor (SCF) and macrophage CSF (M-CSF) for 5 to 9 days before measuring the fraction of cells with a CD14 ${ }^{+} \mathrm{CD} 16^{-}$ or a $\mathrm{CD} 14^{+} \mathrm{CD} 16^{+}$phenotype (Fig. $4 \mathrm{c}$ and Supplementary Figure 6C, D). Compared to sh-SCR-transduced cells, those overexpressing hsa-miR-150 generated more $\mathrm{CD} 14^{+} \mathrm{CD} 16^{+}$monocytes, while those transduced with sh-miR-150 (miR-150 depletion) showed the opposite effect (Fig. 4d).

Hypermethylation of a specific MIR150 gene promoter in CMML. In search for the mechanism driving hsa-miR-150 downregulation in CMML monocytes, we first checked MIR150 coding sequence in sorted peripheral blood monocyte DNA collected from 49 CMML patients ${ }^{24}$ and did not observe any mutation, nor we detected any deletion in 19q13.33 region where MIR150 gene is located. We then analyzed hsa-miR-150 expression in monocytes of 15 CMML patients before and after three to six cycles of the demethylating agent decitabine. While the expression of hsamiR-150 remained low in nine non-responding patients, the six responders demonstrated an increased expression of hsa-miR-150 upon therapy (Fig. 5a), which correlated with the restoration of a normal monocyte subset repartition (Fig. 5b). These results suggested a potential epigenetic down-regulation of MIR150 gene expression in classical monocytes of CMML patients.

MicroRNA expression depends on lineage-specific promoters and enhancers. The $50 \mathrm{~kb}$ region located upstream of MIR150 coding sequence includes seven putative start site regions ${ }^{39}$. In silico analysis of chromatin immunoprecipitation-sequencing (ChIP-seq) data generated by immunoprecipitating $\mathrm{H} 3 \mathrm{~K} 27 \mathrm{me} 3$, $\mathrm{H} 3 \mathrm{~K} 4 \mathrm{me} 1$, $\mathrm{H} 3 \mathrm{~K} 4 \mathrm{me} 3$, and $\mathrm{H} 3 \mathrm{~K} 27 \mathrm{ac}$ in sorted $\mathrm{CD}^{+}{ }^{+} \mathrm{T}$ lymphocytes, $\mathrm{CD} 14^{+}$monocytes, $\mathrm{CD} 56^{+} \mathrm{NK}$ cells, and $\mathrm{CD} 19^{+}$ B lymphocytes indicated distinct histone mark profiles in MIR150 promoter regions of $\mathrm{B}, \mathrm{T}$, and $\mathrm{NK}$ cells compared to monocytes (Fig. 5c). H3K27ac and H3K4me3 marks suggested that the promoter region 1 (R1) was active in T, B, and NK cells, but not in monocytes in which these marks overlapped in a distinct, CpG-enriched region called region 3 (R3). CpG enrichment combined with H3K4me3 and H3K27Ac marks suggested that R3 

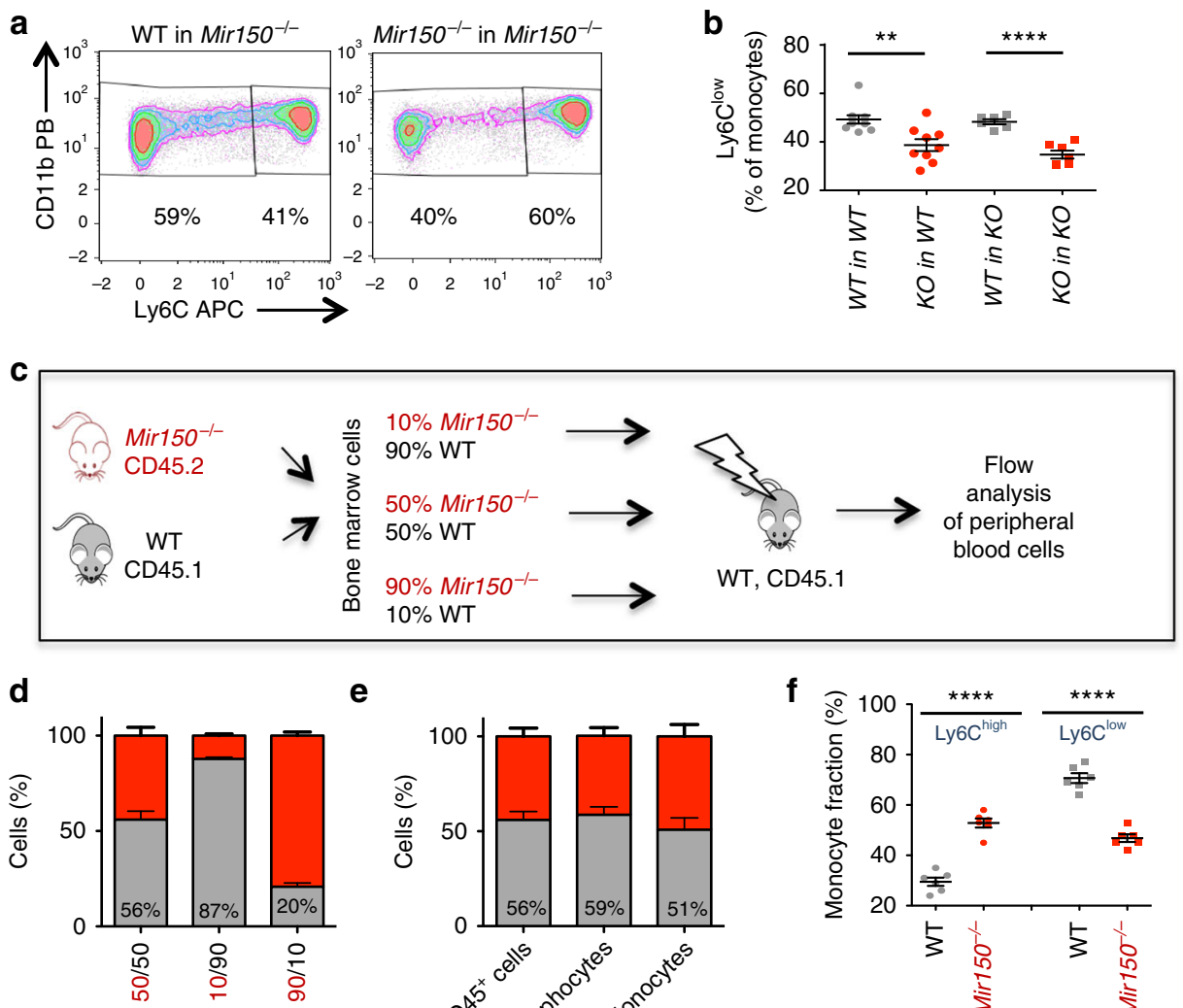

e
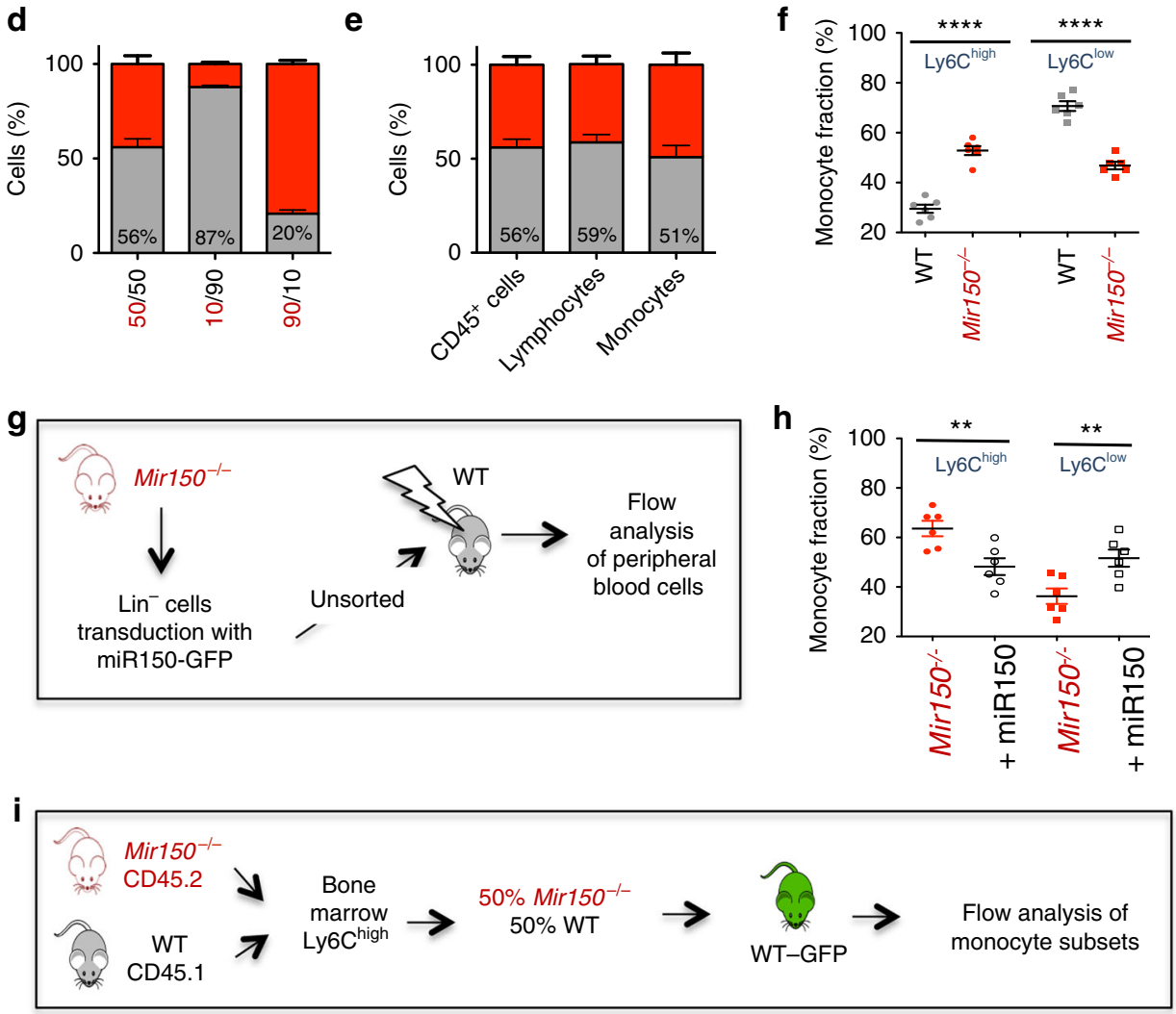

j
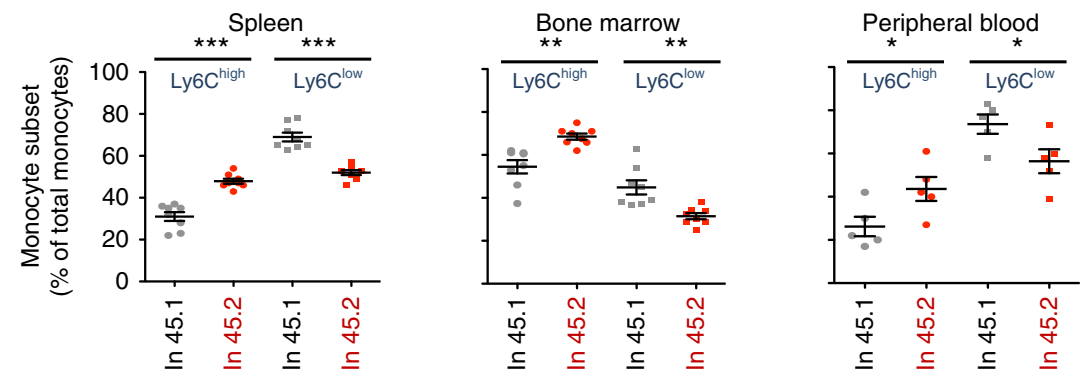

was a promoter with ongoing transcription ${ }^{40,41}$. GRO-cap analyses (GSE60456 accession) identified a distinct transcriptional start site (TSS) on the minus DNA strand in K562 myeloid cells and GM12878 B cells, the TSS being located on R3 in K562 cells (Supplementary Figure 7A). ChIP-seq experiments confirmed that H3K4me3 mark was located on R3 in healthy donor monocytes as well as in those collected from CMML patients (Fig. 5d; peak calling with a $P$ value of $0.05: 107,503,119,009$, and 
Fig. 3 A cell-autonomous effect of miR-150 inactivation in mouse monocyte subset generation. a Representative flow cytometry analysis of peripheral blood monocyte subsets in Mir150-/- recipient mice 6 weeks after engraftment with either wild-type (WT) or Mir150-/- bone marrow cells. b Flow cytometry quantification of Ly6Clow monocytes in the peripheral blood of Mir150-/- $(\mathrm{KO})$ and WT lethally irradiated recipient mice, 6 weeks after transplantation of Mir150-/- or WT bone marrow cells. At least six mice per group. Mean \pm SEM, unpaired $t$ test, ${ }^{\star \star} P<0.01$; ${ }^{\star \star \star \star} P<0.0001$. c Schema of competitive recovery assay using CD45.2+ Mir150-/- and CD45.1 ${ }^{+}$WT bone marrow cells. d Fraction of CD45.1 ${ }^{+}$(gray) and CD45.2+ (red) cells among CD $45^{+}$peripheral blood cells analyzed 8 weeks after competitive transplantation at indicated ratio $(N=6$ per group, mean $\pm /-S E M)$. e Expression of CD45.1 and CD45.2 in indicated cell subsets measured 8 weeks after competitive transplantation at a 50/50 ratio ( $N=6$ per group, mean \pm SEM).

f Monocyte subset repartition among $\mathrm{CD} 45.1^{+}$(gray) and $\mathrm{CD} 45.2^{+}$(red) cells analyzed 8 weeks after competitive transplantation of a $50 / 50$ mixture of CD45.1+ WT and CD45.2+ Mir150-/- bone marrow cells. Mean \pm SEM, paired $t$ test, ${ }^{\star \star \star \star \star} P<0.0001$. $\mathbf{g}$ Schema of in vivo rescue of miR-150 expression. $\mathrm{Lin}^{-} \mathrm{KO}$ bone marrow cells were transduced with a vector expressing both mmu-miR-150 and GFP before transplantation of unsorted cells in WT animals. h Monocyte subset repartition of Mir150-/- and +miR-150 (mmu-miR-150 overexpression) cells was measured 8 weeks later in peripheral blood of chimera animals. Mean \pm SEM, paired $t$ test, ${ }^{\star \star} P<0.01$. i Schema of monocyte transfer experiments. WT-CD45.1 ${ }^{+}$Ly6Chigh and Mir150 $-/-$-CD 45.2+Ly6Chigh bone marrow monocytes were mixed at a 1:1 ratio and injected into GFP-expressing mice. GFP- monocytes were analyzed $40 \mathrm{~h}$ later. j Quantification of 45.1 and 45.2 monocyte subsets in the spleen $(N=8)$, the peripheral blood $(N=5)$, and the bone marrow $(N=8)$ analyzed in three independent experiments. Mean $\pm \mathrm{SEM}$, paired $t$ test, ${ }^{\star} P<0.05 ;{ }^{\star \star} P<0.01 ;{ }^{\star \star \star} P<0.001$

144,139 in control samples, and 64,730, 79,133, 134,677, and 290,419 in CMML samples) with an equal enrichment in control and CMML samples (Supplementary Figure 7B). To validate that R3 could be an active MIR150 promoter, we used CRISP-Cas9 technology to delete a part of R3 region in U937 and K562 myeloid cell lines (Supplementary Figure 7C, D). Compared to wild-type clones, clones in which $\mathrm{R} 3$ has been partially deleted showed a down-regulation of hsa-miR-150 expression without any change in FCGRT gene expression whose TSS is close to $\mathrm{R} 3$ sequence, further supporting a role for R3 as a MIR150specific promoter in these cells (Fig. 5e, f, Supplementary Figure $7 F, G)$.

Bisulfite sequencing of MIR150 R3 sequence detected an increased methylation in CMML patient monocytes compared to young and age-matched healthy donor monocytes (Fig. 6a, Supplementary Figure 8A, B). This methylation decreased in CMML patients who responded to the demethylating agent decitabine, but not in non-responders (Fig. 6b). Again, as FCGRT gene TSS is close to R3 sequence, we used qPCR to check that the expression of this gene remained unchanged in CMML patient compared to healthy donor monocytes (Fig. $6 \mathrm{c}$ and Supplementary Figure $8 \mathrm{C}$ ). We did not detect any methylation of $\mathrm{R} 1$ in monocytes and in T cells of healthy donors and CMML patients, respectively, and the decreased expression of hsa-miR-150 observed in CMML patient monocytes (Fig. 6d and Supplementary Figure $8 \mathrm{D}$ ) was not observed in their T lymphocytes (Fig. 6e and Supplementary Figure 8E). Together, these data designate R3 as an active MIR150 promoter region in healthy monocytes and describe its methylation in CMML patient monocytes as a cause of hsa-miR-150 down-regulation.

We also compared R3 methylation in sorted classical, intermediate, and nonclassical healthy donor monocytes, which showed that the differential expression of miR-150 among these subsets did not depend on R3 methylation (Fig. 7a). ChIP-seq experiments did not detect any difference in $\mathrm{H} 3 \mathrm{~K} 4 \mathrm{mel}$, $\mathrm{H} 3 \mathrm{~K} 27 \mathrm{Ac}$, and H3K4me3 marks at R3 among monocyte subsets (Fig. 7b, c and Supplementary Figure 9A) ${ }^{42}$. Interestingly, while the expression of miR-150 was decreased in sorted classical and intermediate monocyte subsets collected from $10 \mathrm{CMML}$ patients before any treatment and compared to healthy donor monocyte subsets, its expression was not decreased in the rare, residual nonclassical monocytes in CMML patients (Fig. 7d, Supplementary Figure 9B, C), suggesting that a fraction of leukemic cells may escape the epigenetic down-regulation of MIR150 gene in these patients.

TET3 is a miR-150 target that regulates monocyte subsets. In order to identify hsa-miR-150 targets in classical monocytes,
microRNA pull-down was performed in sorted cells, $20 \mathrm{~h}$ after their transfection with biotinylated hsa-miR-150. Among the RNA sequences collected from four independent experiments (Supplementary Table 2) and predicted to be hsa-miR-150 targets using TargetScan algorithm, we selected TET3 as it was also confirmed to be a potential miR-150 target using RNA-hybrid software, providing a score of -29.2 and $-30.8 \mathrm{kcal} / \mathrm{mol}$ in human and mouse, respectively (Fig. 8a). TET3 had been recently identified as a hsa-miR-150 target in acute myeloid leukemia cells ${ }^{43}$. These results were confirmed in U937 cells (Supplementary Figure 10A). Real-time quantitative PCR (RT-qPCR) experiments detected a decreased expression of TET3 in monocytes generated by liquid culture of $\mathrm{CD} 34^{+}$cells transduced with a hsa-miR-150-expressing lentivirus, using cells transduced with a scrambled RNA as a control (Fig. 8b and Supplementary Figure 10B). Immunoblot analysis of classical monocytes sorted from CMML peripheral blood samples showed an increased expression of TET3 protein when compared to healthy donor classical monocytes (Fig. 8c). RNA-sequencing of classical and nonclassical monocyte subsets of four healthy donors identified 2176 differentially expressed genes with an adjusted $P$ value $<0.01$ (Supplementary Table 3 and Supplementary Figure 10C), including TET3 whose expression was down-regulated in nonclassical monocytes $(-1.6$-fold, $P=0.001)$ (Fig. $8 \mathrm{~d}$ ). Finally, an abnormal repartition of monocyte subsets, with an increase in Ly6C ${ }^{\text {low }}$ monocytes at the expanse of Ly $6 C^{\text {high }}$ cells, was detected in the blood of mice carrying inactivated Tet 3 alleles compared to wild-type littermates (Fig. 8e, f and Supplementary Figure $10 \mathrm{D}-\mathrm{F})$, without any change in the mean fluorescence intensity of CD115 and CX3CR1 at the surface of Tet $3^{-1-}$ mouse monocytes (Supplementary Figure 10G). Hence, these experiments argued for TET3 as a target of miR-150 whose down-regulation is required for the differentiation of classical into nonclassical monocytes, in mice and in humans.

To further explore how disruption of the miR-150/TET3 axis could alter the fate of monocytes, we sequenced RNA in Ly6Chigh and Ly6Clow monocytes collected from Mir $150^{-1-}$ and Tet $3^{-1-}$ mice and their wild-type littermates. Comparison of gene expression in Ly6Chigh and Ly6C $\mathrm{C}^{\text {low }}$ monocytes collected from the two series of wild-type littermates confirmed that Tet 3 gene expression was significantly lower in Ly6C low monocytes, together with Ccr2 and $L y 6 c$ genes (Fig. 8g), further validating the decreased expression of TET3 gene in nonclassical compared to classical human monocytes (Fig. 8d). Compared to wild-type cells, 127 and 78 genes were differentially expressed in Mir150-/Ly6Chigh and Ly6C Cellow, respectively (Fig. 9 and Supplementary Table 4). Of note, Mir150-1- gene deletion did not significantly change the expression of Cd115, Cebpb, Nr4a1, and 

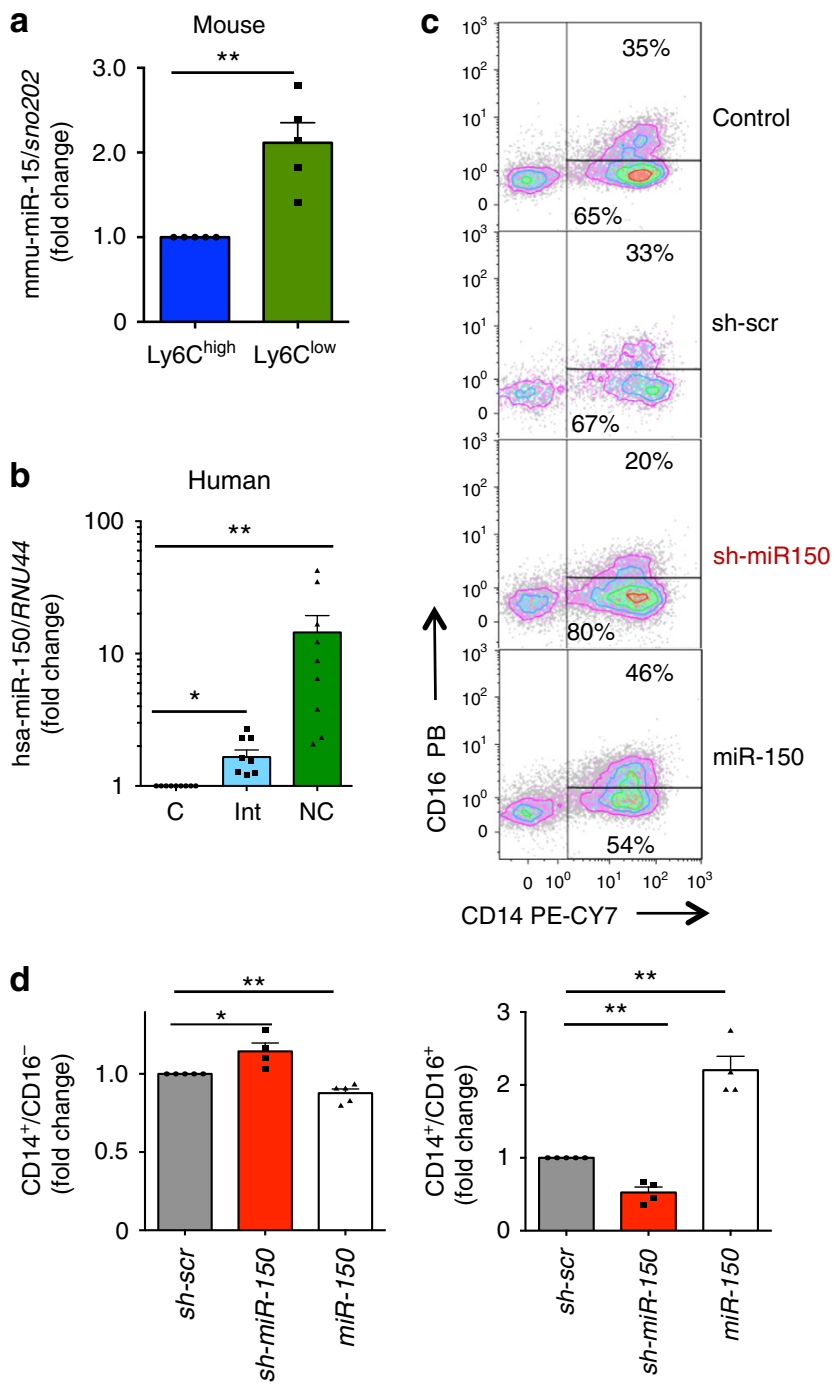

Fig. 4 Differential expression of miR-150 in human and mouse monocyte subsets. a qRT-PCR analysis of mmu-miR-150 expression normalized to sno202 in Ly6Clow compared to Ly6Chigh monocytes sorted from wild-type mice peripheral blood. Results are expressed as fold-change relative to Ly6Chigh monocytes. Mean \pm SEM of five independent experiments, paired $t$ test, ${ }^{\star \star} P<0.01$. b qRT-PCR analysis of hsa-miR-150 expression normalized to RNU-44 in peripheral blood classical (C), intermediate (Int), and nonclassical (NC) monocytes sorted from peripheral blood samples of nine healthy donor controls. Results are expressed as fold-change relative to classical monocytes. Mean \pm SEM; paired $t$ test, ${ }^{\star} P<0.05$; ${ }^{\star \star} P<0.01$. c, d Sorted $\mathrm{CD}_{3}{ }^{+}$cells were not transduced (control) or transduced with vectors expressing the GFP and a control shRNA (Sh-SCR) or a shRNA targeting hsa-miR-150 (sh-miR-150) or hsa-miR-150 (miR-150), and then cultured in liquid medium for 7 days in the presence of SCF and M-CSF before flow cytometry analysis of generated monocyte subsets, based on CD14 and CD16 expression. c shows a representative flow cytometry analysis. d shows the fraction of $\mathrm{CD}_{14}{ }^{+} \mathrm{CD} 16^{-}$(left panel) and $\mathrm{CD} 14^{+} \mathrm{CD} 16^{+}$(right panel) generated by $\mathrm{CD}_{3}{ }^{+}$cells with decreased (sh-miR-150) or increased (miR-150) hsa-miR-150 level relative to controls (sh-scr). Mean \pm SEM of four independent experiments, paired $t$ test, ${ }^{\star} P<0.05 ;{ }^{\star \star} P<0.01$

Tet3 genes (Supplementary Table 3). This latter result could indicate that miR-150 targets Tet3 translation rather than Tet3 gene expression. Pathway analysis using Ingenuity software indicated most significant changes in cell migration $(z$-score $-1.98, P$ value $\left.2.76 \times 10^{-5}\right)$ and immune cell death $(z$-score -2.13 ,
$P$ value $6.6 \times 10^{-3}$ ) pathways in Ly6Chigh cells, whereas genes involved in proliferation $\left(z\right.$-score $-2.6, P$ value $\left.2.88 \times 10^{-2}\right)$ were altered in Ly6C ${ }^{\text {low }}$ cells. Analysis of gene expression in $\mathrm{Tet}^{-1-}$ cells detected the higher number of differentially expressed genes was in Tet3 ${ }^{-1-}$ Ly6C ${ }^{\text {low }}$ cells $(1019$ genes; Fig. 9 and Supplementary Table 4), further suggesting an important role of TET3 in the generation or functions of this monocyte subset.

\section{Discussion}

While the diversity and versatility of circulating monocytes is well recognized, the molecular mechanisms driving the generation of minor monocyte subsets, including Ly6 $\mathrm{C}^{\mathrm{low}}$ monocytes in mice and $\mathrm{CD} 16^{+}$monocytes in humans, are only partly depicted. The present study identifies a role for miR-150-mediated regulation of TET3 expression in the generation of these monocyte subsets. hsa-miR-150 expression in monocytes is shown to be regulated by a lineage-specific promoter whose hypermethylation in CMML cells is associated with a decrease in the fraction of circulating intermediate and nonclassical monocytes. The deletion of either Mir150 or Tet3 gene is associated with an abnormal repartition of monocyte subsets in mouse models.

The expression of hsa-miR-150 is deregulated in several hematopoietic malignancies ${ }^{44}$, that is, in the most abundant miRNA detected in chronic lymphocytic leukemia B cells in which it modulates $B$ cell receptor signaling 39 while being downregulated in a variety of human non-Hodgkin lymphoma cells, for example, in anaplastic large-cell lymphomas in which restoration of its expression through the use of hypomethylating drugs delays disease progression in xenograft models ${ }^{45}$. A decreased expression of hsa-miR-150 has been also described in chronic $^{46}$ and acute ${ }^{47}$ myeloid malignancies, for example, hsamiR-150 maturation is inhibited and its expression is downregulated in acute myeloid leukemias involving the mixed lineage leukemia $(M L L)$ gene ${ }^{47}$. We detected hsa-miR-150 as the most abnormally expressed miRNA in sorted circulating monocytes of patients with a CMML, which are mostly classical monocytes, and we identified the hypermethylation of a lineage-specific promoter as the mechanism for its decreased expression. While Mir150 deletion in mice was not sufficient to mimic all the features that define CMML phenotype, the abnormal repartition of monocyte subsets in these animals was reminiscent of that observed in CMML patients ${ }^{20,26}$. This observation suggested a role for miR150 in the generation of nonclassical monocytes.

miR-150 has been involved in the development of lymphoid and myeloid lineages ${ }^{44}$. This microRNA plays a critical role in $\mathrm{B}$ cell development through directly regulating the expression level of c-Myb transcription factor ${ }^{30,48}$, and promotes NK cell development and maturation ${ }^{32}$. hsa-miR-150 also drives the differentiation of myeloid progenitors toward megakaryocytes at the expense of erythroid cells ${ }^{34}$, and regulates hematopoietic recovery after injury with chemotherapeutic drugs ${ }^{35}$. Among circulating monocytes, the expression of miR-150 was observed to be lower in classical than in nonclassical monocytes ${ }^{49,50}$, with some discrepancies regarding intermediate monocytes ${ }^{51}$ that could be related to the heterogeneity of this subset ${ }^{52}$. The abnormal repartition of monocyte subsets in the peripheral blood of Mir150 -I- mice led us to identify a cell-autonomous function of this microRNA in the generation of Ly6C Clow monocyte subset.

Accumulating evidence supports that, in the steady state, most if not all mouse $\mathrm{Ly} 6 \mathrm{C}^{\mathrm{low}}$ and human $\mathrm{CD} 14^{-} \mathrm{CD} 16^{+}$monocytes derive from $\mathrm{Ly} 6 \mathrm{C}^{\text {high }}$ and $\mathrm{CD} 14^{+} \mathrm{CD} 16^{-}$monocytes, respectively ${ }^{16,53-56}$. Deletion of the Nur77/Nr4a1 gene demonstrated the requirement of the encoded transcription factor for the development and survival of Ly6C $\mathrm{C}^{\text {low }}$ monocytes ${ }^{12}$, which was further confirmed by deletion of a unique sub-domain within the 
a

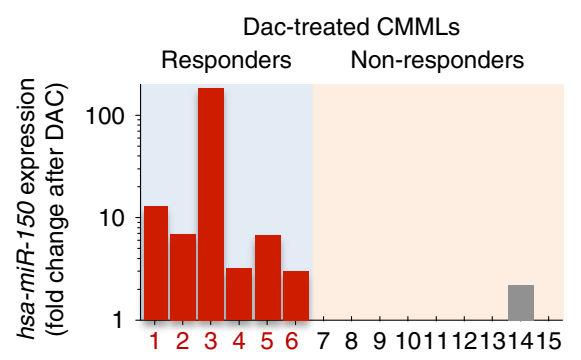

b

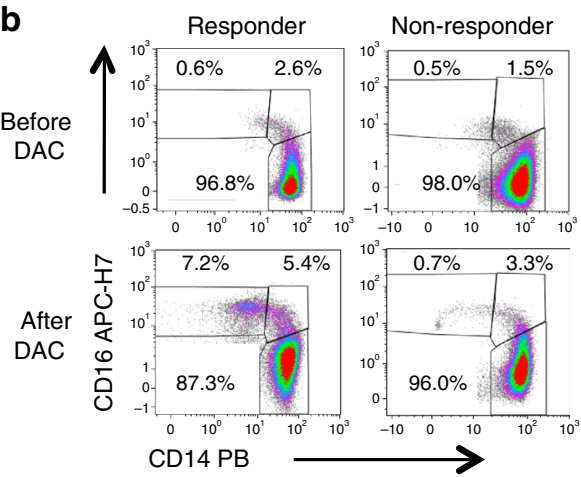

e U937-CRISPR-R3

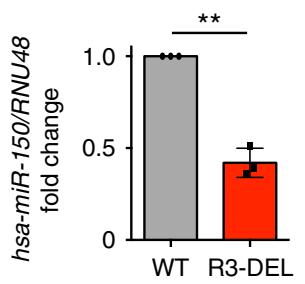

f K562-CRISPR-R3

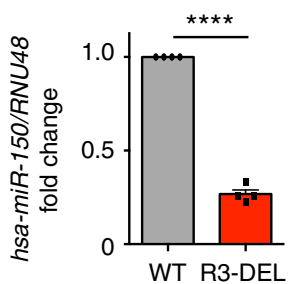

C

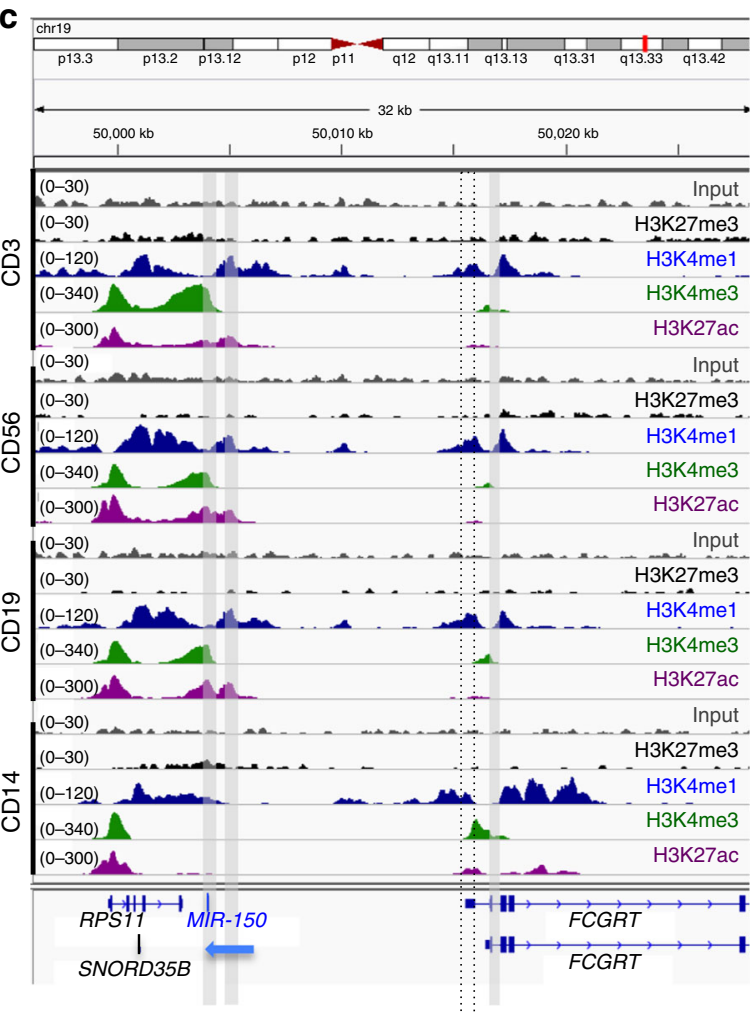

R1 R2

MIR150 TSS

d

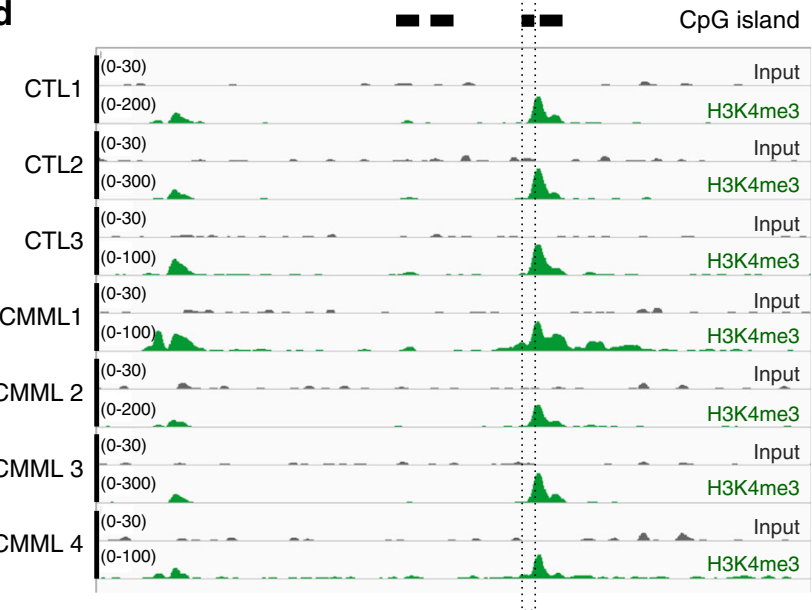

Fig. 5 Regulation of hsa-miR-150 expression by a monocytic lineage-specific promoter. a Expression of hsa-miR-150 (normalized to RNU-6B) in monocytes of 15 CMML patients according to their response to three to six cycles of decitabine (DAC). Results are expressed as hsa-miR-150 expression fold-change after versus before treatment. $\mathbf{b}$ Representative dot plots showing the monocyte subset repartition before and after treatment with decitabine (DAC) in a responder and a non-responder CMML patient. c Input (gray, scale 0-30) and ChIP-seq data for H3K27me3 (black, scale 0-30), H3K4me1 (blue, scale 0-120), H3K4me3 (green, scale 0-340) and H3K27ac (purple, 0-300) obtained in human CD3 $+, \mathrm{CD}_{19+}^{+}, \mathrm{CD}_{56}{ }^{+}$, and CD14 ${ }^{+}$cells were collected from www.roadmapepigenomics.org/. miR-150 promoters are indicated as R1, R2, R3, and R4. Black rectangles indicate CpG islands. MIR150 gene (blue) is on DNA minus strand (blue arrow), while other genes (in black) are on the DNA plus strand. $\mathbf{d}$ Input (gray) and ChIP-seq of H3K4me3 (green) in sorted CD14 ${ }^{+}$ cells collected from three healthy donors (CTL) and four CMML patients. Data are shown as normalized Bigwig. Scales are on the left. e, $\mathbf{f}$ Expression of hsa-miR-150 (normalized to RNU-48) in U937 (e) and K562 (f) clones in which R3 has been partially deleted using a CRISPR/Cas9 gene editing method. Results are expressed as hsa-miR-150 expression fold-change in R3-deleted clone (R3-DEL, red bars) relative to wild-type clone (WT, gray bars, normalized to 1). Results are mean \pm SD of a minimum of three independent experiments. Paired $t$ test, ${ }^{\star \star} P<0.01 ;{ }^{\star \star \star \star} P<0.0001$

Nr4a1 gene enhancer that is specifically required for Ly6Clow subset development, without altering $\mathrm{Nr} 4 \mathrm{a} 1$ expression in tissue macrophages $^{13}$.

Another transcription factor, CCAAT/enhancer-binding protein $\beta(\mathrm{C} / \mathrm{EBP} \beta)$, was recently shown to play a critical role in Ly6C low monocyte survival ${ }^{36}$. The deletion of $\mathrm{C} / \mathrm{EBP} \beta$ could down-regulate the expression of Csf1r (also known as Cd115 and $\mathrm{M}$-CSFactor receptor) at the monocyte surface. CSF1R genetic deletion, pharmacological inhibition ${ }^{57,58}$, and constitutive mutation $^{17,59}$ all decrease the generation of nonclassical monocyte subsets. The chemokine receptor CX3CR1, which is highly expressed on nonclassical monocytes ${ }^{60}$, and the G-coupled 
a
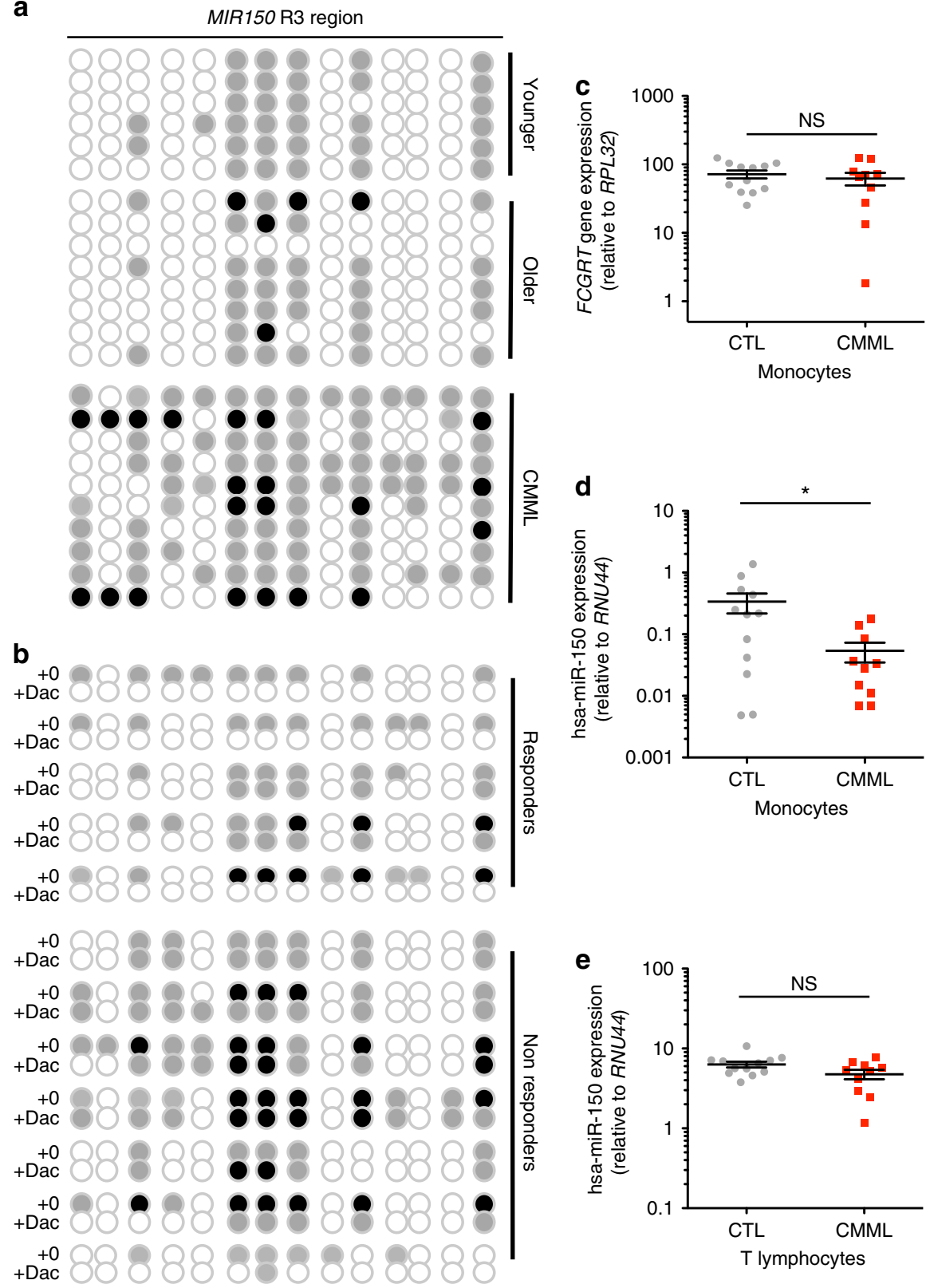

Fig. 6 Methylation of MIR150 region 3 in CMML classical monocytes. a DNA methylation of MIR150 region 3 (R3) was analyzed in sorted CD14+ peripheral blood monocytes from six young (<65 years old) and 8 older ( $>65$ years old) healthy donors and $10 \mathrm{CMML}$ patients. White ball: no methylation; gray ball: methylated on one strand; black ball: methylated on two strands. b DNA methylation status of R3 was analyzed in sorted CD14 ${ }^{+}$monocytes from 12 CMML patients before $(+0)$ and after ( + Dac) decitabine treatment, including five responders and seven non-responders. White ball: no methylation; gray ball: methylated on one strand; black ball: methylated on two strands. c-e qRT-PCR analysis of FCGRT and hsa-miR-150 expression in sorted cells collected from 12 healthy donors (CTL) and 10 CMML patients. Samples are those studied by bisulfite sequencing. FCGRT gene expression is normalized to RPL32 in sorted monocytes (c), hsa-miR-150 expression is normalized to RNU-44 (d) in sorted monocytes and in CD3+ T lymphocytes (e). Mean \pm SEM, unpaired $t$ test: ${ }^{\star} P<0.05$. NS, nonsignificant

receptor for sphingosine-1 phosphate, S1PR5 ${ }^{61}$, were also suggested to regulate the fraction of circulating nonclassical monocytes. Our results identify miR-150 as an additional molecular regulator of nonclassical monocyte generation. The consequences of miR-150 down-regulation may be independent of Nr4a1, C/ $\mathrm{EBP} \beta$, and CSF1R, as a decrease in its expression affects the generation of nonclassical monocytes without altering their survival. Having identified the lineage-specific R3 promoter that regulates hsa-miR-150 expression in mature monocytes, a next step will be to depict the transcription factors that interact with this sequence to promote hsa-miR-150 up-regulation along the generation of nonclassical monocytes.

While c-Myb is a direct and essential miR-150 target in B lymphocytes and megakaryocytes, we failed to identify any link between miR-150 and c-Myb expression levels in monocytes. Similarly, miR-150 could regulate effector $\mathrm{CD}^{+} \mathrm{T}$ cell functions independently of $\mathrm{c}-\mathrm{Myb}^{31}$. In order to identify hsa-miR-150 targets in monocytes, we performed microRNA pull-down as this method allows identifying RNAs whose either stability or translation is altered by the studied microRNA. Among the predicted 
a
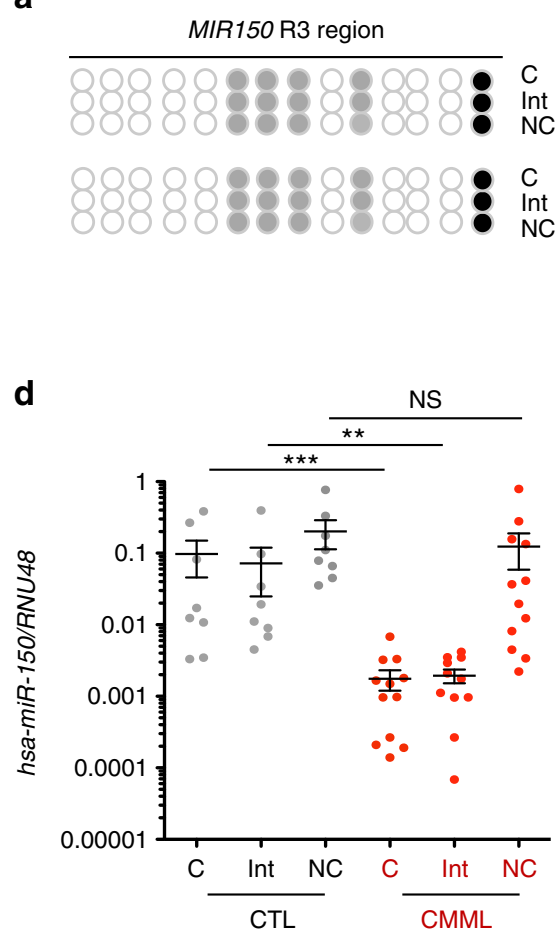

b C
Int
NC
C
Int
NC
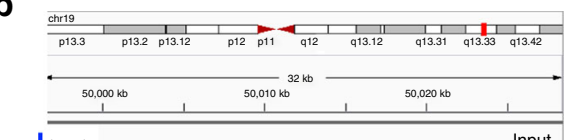

C

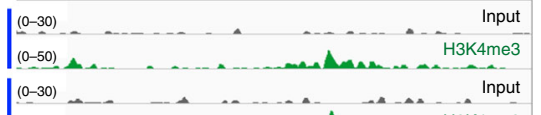

C $\mid \begin{aligned} & (0-30) \ldots \ldots \ldots \ldots \\ & (0-120)\end{aligned} \ldots \ldots$ H3K4me3

Int $\mid \begin{array}{lll}(0-30) \\ (0-220)\end{array} \ldots \ldots$

Int $\mid \frac{(0-30)}{(0-120)} \ldots$

NC

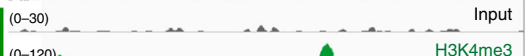

NC

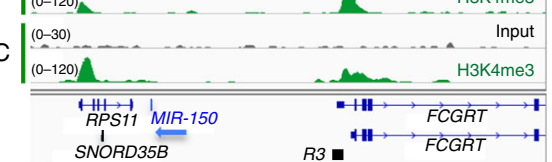

C

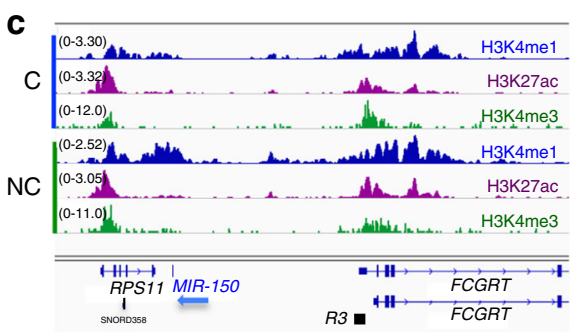

Fig. 7 Methylation of MIR150 region 3 in human monocytes subsets. a DNA methylation of MIR150 region 3 (R3) was analyzed in classical (C), intermediate (Int), and nonclassical (NC) monocytes sorted from healthy donor peripheral blood samples. (Results of two healthy donors are shown.) White ball: no methylation; gray ball: methylated on one strand; black ball: methylated on two strands. b. Input and ChIP-seq of H3K4me3 in sorted classical (C), intermediate (Int), and nonclassical (NC) monocytes collected from two healthy donors. Data are shown as normalized Bigwig. R3 region is representated. c ChIP-seq data for H3K4me1 (blue) and H3K27ac (purple) collected from ref. ${ }^{42}$ and for H3K4me3 (green), conducted in human classical (C) and nonclassical (Non-C) monocytes. R3 region is representated. d qRT-PCR analysis of hsa-miR-150 expression normalized to RNU-48 in classical (C), intermediate (Int), and nonclassical (NC) monocytes sorted from peripheral blood samples of 8 healthy donor controls (CTL) and $12 \mathrm{CMML}$ patients. Mean \pm SEM; Mann-Whitney test: ${ }^{\star \star} P<0.01$; ${ }^{\star \star \star} P<0.01$. NS nonsignificant

targets containing evolutionary conserved binding sites for miR150 , TET3 was of particular interest. The three TET proteins are dioxygenases that utilize $\mathrm{Fe}(\mathrm{II})$ and 2-oxoglutarate as cofactors to catalyze the oxidation of 5-methylcytosine into 5hydroxymethylcytosine and other derivatives, thereby regulating DNA methylation ${ }^{62}$. Whereas TET2 mutations are commonly detected in myeloid malignancies, especially in CMML, and mutations in TET3 are very rare $^{63}$ and late events in clonal evolution $^{24}$. MicroRNAs regulate the expression level of TET enzymes in a cell-specific manner ${ }^{64}$, for example, hsa-miR-15b regulates TET3 expression during neurogenesis ${ }^{65}$. TET3 has been recently validated as a miR-150 target in acute myeloid leukemia cells ${ }^{43}$. The increased fraction of Ly6 $6 C^{\text {low }}$ monocytes detected in the blood of Tet $3^{-1-}$ mice compared to wild-type littermates, and observations made in healthy donor and CMML patient cells, support a role for TET3 as a miR-150 target whose downregulation is required for the optimal generation of nonclassical monocytes.

The abnormal repartition of monocyte subsets that characterizes CMML has provided a diagnostic tool in patients with a monocytosis to rapidly distinguish this myeloid malignancy from a reactive monocytosis ${ }^{20}$. This characteristic feature also provides new insights into circulating monocyte biology by identifying the role of a miR-150/TET3 axis in the development of nonclassical monocyte subsets. Egression of classical monocytes from the bone marrow requires expression of the chemokine receptor CCR2 ${ }^{3}$. These classical monocytes transit to the blood in which their halflife at steady state was demonstrated to be one day or less, in mice 55,66 and in humans ${ }^{16}$. Most of these classical monocytes subsequently leave the circulation or die, whereas a small fraction of them give rise to intermediate and nonclassical monocytes and demonstrate a longer lifespan ${ }^{16,55}$. It remains unclear how classical monocytes are selected to differentiate into nonclassical monocytes rather than migrating into tissues. Recent investigations in mice suggested that Notch-2 signaling activated by endothelial cells was required for cell fate decision, that is, to promote classical Ly6 $\mathrm{C}^{\text {high }}$ conversion into Ly6 $\mathrm{C}^{\text {low }}$ monocytes ${ }^{67}$. These results suggest a scenario in which Notch-2 signaling would promote the expression of miR-150 and the modulation of downstream targets such as TET3. Deciphering in human miR150 promoter regulation by key transcription factors remains to be explored.

In conclusion, our data delineate a miR-150/TET3 axis involved in the generation of nonclassical monocytes. Targeting of this pathway provides strategies to modulate monocyte subset repartition and investigate their therapeutic interest in pathological situations.

\section{Methods}

Healthy donor and patient samples. Buffy coats collected from male and female healthy donors (Etablissement Français du sang, Rungis, France) and peripheral blood samples collected on ethylene diaminetetraacetic acid (EDTA) from healthy volunteers were used to sort control cells. A learning cohort of CMML (Supplementary Table 1) was made of patients enrolled between November 2008 and June 2009 in a previously reported phase 2 clinical trial (GFM-DEC-LMMC-2007-02) ${ }^{68}$. We also get access to sorted blood monocytes collected after three to six cycles of decitabine treatment in 15 of the patients enrolled in this trial, including six 
a

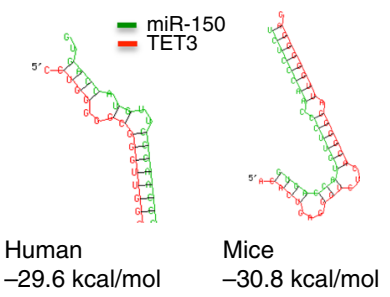

C

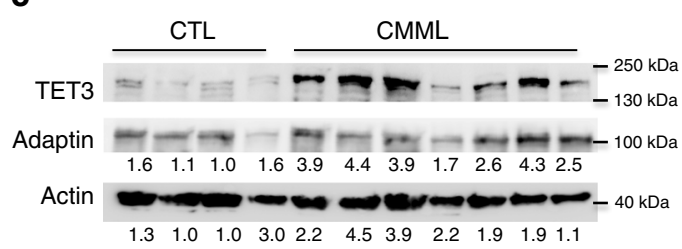

e

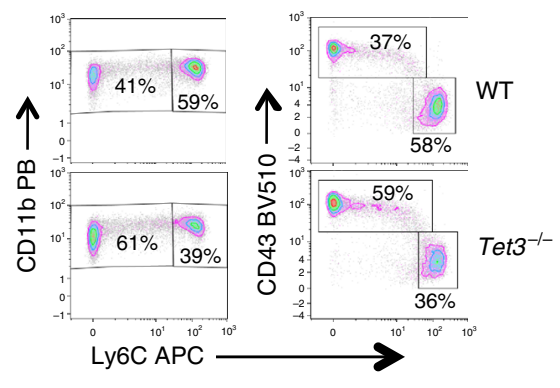

g

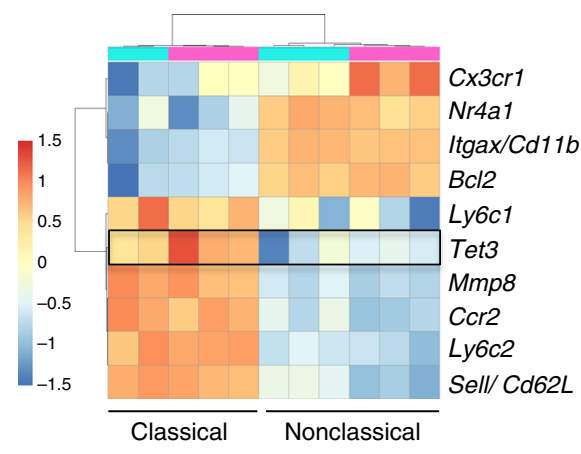

b

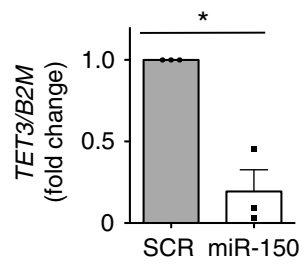

d
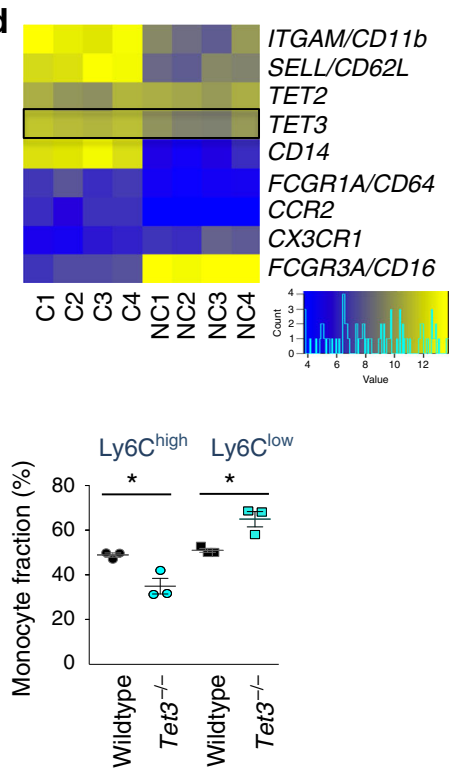

WT littermates of $\mathrm{Mir} 15 \mathrm{O}^{-/}$mice

WT littermates of $T e 3^{-/}$mice

Fig. 8 TET3 is a miR-150 target involved in monocyte subset differentiation. a Hybridization analysis of 3'-UTR of TET3 with mature sequences of miR-150 using RNA-hybrid software in human and mouse. Structures with minimum free energy are shown. $\mathbf{b}$ Expression of TET3 (normalized to B2M) in monocytes generated by culture of human CD34+ cells transduced with hsa-miR-150-GFP compared to control-transduced cells (SCR). Results are expressed as TET3 expression fold-change in hsa-miR-150-overexpressing cells relative to control (SCR, normalized to 1). Mean \pm SEM of three independent experiments. Paired $t$ test, ${ }^{\star} P<0.05$. c Immunoblot analysis of TET3 expression in four healthy donor (CTL) and seven CMML patient monocytes. ADAPTIN and ACTIN are the loading controls. TET3/ADAPTIN or TET3/ACTIN ratio are shown below. Blots have been cropped for clarity. d Heat map of TET2 and TET3 gene expression, compared to a selection of differentially expressed genes in classical and nonclassical monocyte subsets as measured by RNA-seq ( $N=4$ healthy donors). The expression of genes characterizing these two subsets is shown. e Representative flow cytometry analysis of CD11b ${ }^{+}$or $\mathrm{CD}_{4} 3^{+}$ monocyte populations gated on Ly6C expression in peripheral blood of a wild-type (WT) and a Tet3-/- (KO) mice. f Monocyte subsets measured by flow cytometry in WT and Tet3 ${ }^{-/}$- mice. $N=3$, mean $\pm \mathrm{SEM}$, unpaired $t$ test, ${ }^{\star} P<0.05$. One representation of three independent experiments is shown. $\mathbf{g}$ Heat map of differentially expressed genes in Ly6Chigh and Ly6Clow monocyte subsets as measured by RNA-seq of two series of wild-type littermates ( $N=3$ wild-type mice for Mir150-/- littermates, and 2 or 3 wild-type mice for Tet $3^{-/-}$littermates)

responders and nine non-responders. A validation cohort (Supplementary Table 1) was made of 139 newly diagnosed CMML patients whose samples were collected with informed consent following the authorization provided by the ethical committee Ile-de-France 1 (DC-2014-2091). Patients were reclassified according to the latest 2016 World Health Organization criteria ${ }^{29}$.

Mouse models. All the animal experiments were approved by the Gustave Roussy ethical review board (2012-018-16-540 and 2016-104-7171). Wild-type CD45.1 and CD45.2, C57BL/6 animals expressing the GFP under human ubiquitin C promoter ${ }^{38}$ and $m i R-150^{-1-}$ mice were purchased from Jackson Laboratories (Charles River France, L'Arbresle, France). Mice harboring Tet3 allele with the coding sequences of exon 11 flanked by two loxP, a strategy similar that described with the Tet2 allele ${ }^{69}$, were generated by the Plateforme Recombinaison homolog (Institut Cochin, Paris, France) and were intercrossed with mice expressing tamoxifen-inducible Cre $\left(\right.$ Cre-ER $\left.{ }^{\mathrm{T}}\right)$ transgene under control of the Scl/Tal1 promoter/enhancer. To delete Tet3-floxed alleles, tamoxifen was solubilized at $20 \mathrm{mg} / \mathrm{ml}$ in sunflower oil (Sigma-Aldrich, SaintQuentin Fallavier, France) and $8 \mathrm{mg}$ tamoxifen were administered to mice once 


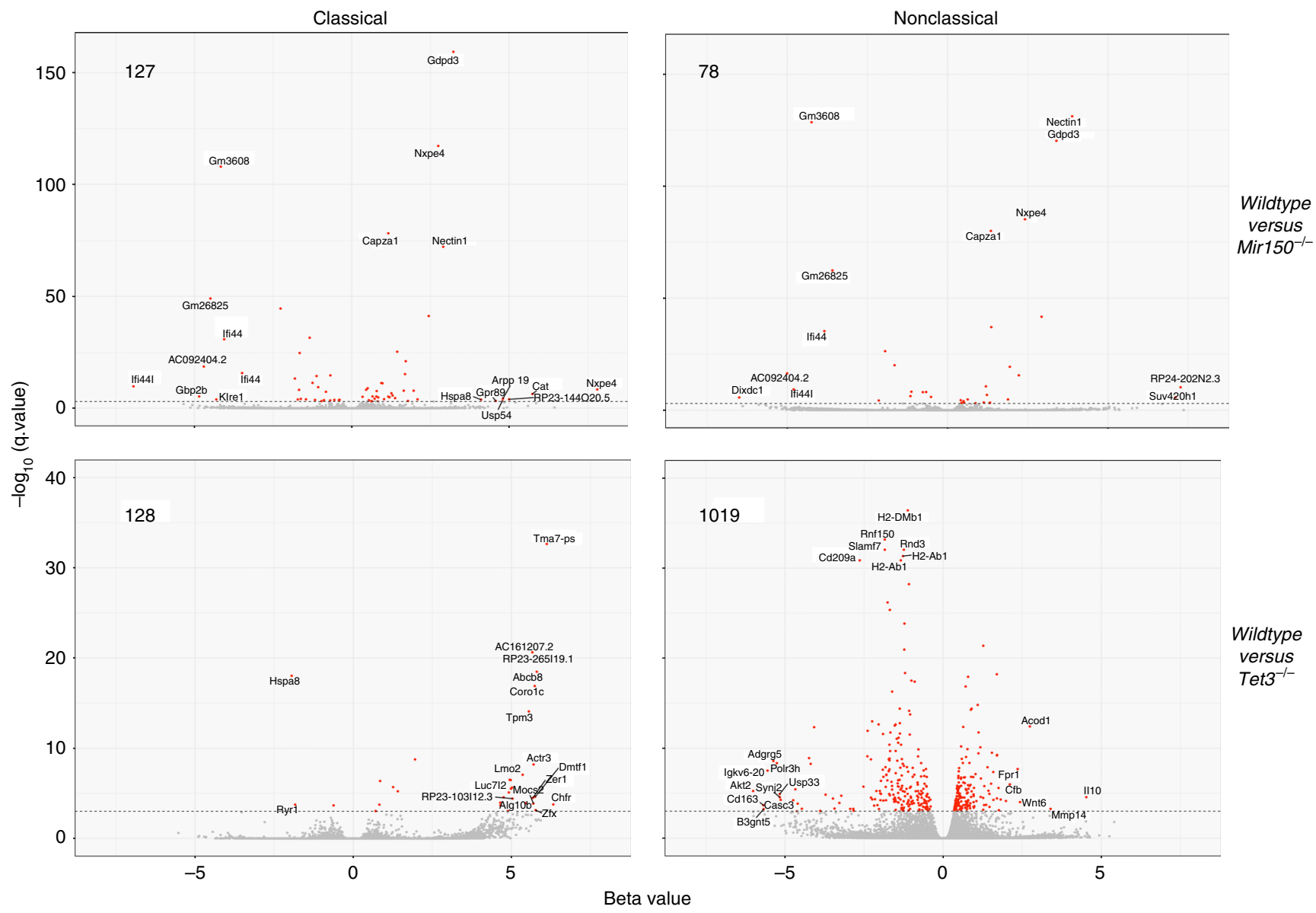

Fig. 9 RNA-sequencing of mouse monocyte subsets. Volcano plot representation of differentially expressed genes in wild-type littermates versus either Mir150-/- (upper panels) or Tet3-/- (lower panels) monocytes subtypes. The number of differentially expressed genes (cut-off $q$ value 0.001 ) is indicated in each panel, as well as the name of differentially deregulated genes

per day for 2 days via oral gavage. For competitive and rescue experiments, recipient mice were housed in a barrier facility under pathogen-free conditions after transplantation. Cell transfer experiments were performed in 8- to 12-week-old female mice.

Human primary cell culture $\left(\mathrm{CD} 34^{+}\right)$and transduction. $\mathrm{CD} 34^{+}$cells were cultured $24 \mathrm{~h}$ at $1 \times 10^{6}$ cells $/ \mathrm{ml}$ in MEM- $\alpha$ medium (Thermo Fisher Scientific) supplemented with $10 \%$ heat-inactivated fetal bovine serum (FBS), 1\% penicillin/ streptomycin and $2 \mathrm{mM}$ L-glutamine (Thermo Fisher), SCF (50 ng/ml), interleukin3 (IL-3, $10 \mathrm{ng} / \mathrm{ml}$ ), IL-6 (10 ng/ml), thrombopoietin (TPO, $10 \mathrm{ng} / \mathrm{ml}$ ), Fms-like tyrosine kinase $3(50 \mathrm{ng} / \mathrm{ml})$, in a $37^{\circ} \mathrm{C}$ incubator with a humidified atmosphere of $5 \% \mathrm{CO}_{2}$ in air. Cells were transduced at a multiplicity of infection (MOI) of 35-75 either with pRRL-EF1hsa-miR-150 or pRRL-H1-anti-miR-150 or pRRL-H1-shCTRL, sorted again $48 \mathrm{~h}$ after transduction using a cell sorter and anti-CD34 antibodies and cultured during 5 to 10 days in complete MEM- $\alpha$ with SCF $(50 \mathrm{ng} / \mathrm{ml})$ and human $\mathrm{M}-\mathrm{CSF}(100 \mathrm{ng} / \mathrm{ml})$. Only transduced-GFP ${ }^{+}$cells were analyzed.

Cell line culture and transfection. The K562 cell line, originally established from the pleural effusion of a patient with chronic myeloid leukemia, and the U937 cell line, established from a histiocytic lymphoma, were purchased from ATCC and were maintained in RPMI- 1640 Glutamax medium supplemented with $10 \%$ heatinactivated FBS, $1 \%$ penicillin/streptomycin, and $2 \mathrm{mM}$ L-glutamine (Thermo Fisher), in a $37^{\circ} \mathrm{C}$ incubator with a humidified atmosphere of $5 \% \mathrm{CO}_{2}$ in air. Cell lines were negative for mycoplasma contamination. Cells were transfected with lentiviral plasmids coding for the two guide RNAs (gRNA471-Cherry and gRNA289-GFP) using Lipofectamine 2000 as recommended by the manufacturer (Invitrogen, Cergy Pontoise, France), sorted after $48 \mathrm{~h}$ by flow cytometry for double-positive cells and cloned at one cell per well. Wild-type clones corresponding to an uncut DNA, heterozygotes and homozygous clones corresponding, respectively, to one or two alleles deleted for the $\mathrm{R} 3$ region.

Antibodies and reagents. H3K4me3 antibody used for ChIP-seq was purchased from Active Motif (La Hulpe, Belgium, no. 39159, dilution $3 \mu \mathrm{l}$ for $100 \mu \mathrm{l}$ ). For immunoblot experiments, we used an anti-TET3 (GTX121453, GeneTex Inc., Clinisciences, Nanterre, France, dilution 1/1000e), an anti-a-ADAPTIN (M-300, Santa Cruz Biotechnology, Clinisciences, dilution 1/1000e) and an anti-ACTIN (A5441, Sigma-Aldrich, dilution 1/5000e) antibodies. Secondary antibodies were purchased at Thermo Fisher Scientific (anti-rabbit, anti-mouse, dilution 1/20,000e). For flow cytometry, antibodies were purchased from BD Biosciences (San Diego, USA), BioLegend (San Diego, USA), and Beckman Coulter (Villepinte, France). Detailed references are in Supplementary Table 5.

DNA and RNA preparation. Genomic DNA and RNA were isolated using Norgen standard procedures, and total RNA from mouse monocytes was extracted using Trizol (Invitrogen, Waltham, MA, USA) and Direct-zol RNA Kit (Zymo Research, Ozyme, France). For microarray, total RNA (100 ng) was extracted using Trizol (Invitrogen, Waltham, MA, USA) from sorted CD14 ${ }^{+}$monocytes of 33 CMML patients and 5 healthy donors. In addition, a pool of control samples was analyzed.

MiRNA preparation and hybridization. RNA samples were dephosphorylated with calf intestine alkaline phosphatase (GE Healthcare Europe GmbH, Velizy Villacoublay, France), denatured with dimethyl sulfoxide, and labeled with pCpCy3 using T4 RNA ligase before RNA hybridization to Agilent human miRNA microarrays v3.0 (Agilent, Les Ulis, France) for $20 \mathrm{~h}$ at $55^{\circ} \mathrm{C}$ under rotation. After washes, microarrays were scanned with an Agilent microarray scanner using high dynamic range settings before extracting data using the Agilent Feature Extraction Software (10.7.3.1). Quantile normalization was made using the normalizeBetweenArray function from R package LIMMA. Data were $\log 2$ transformed. When several probes were spotted for a same miRNA, the median of all probes was averaged. Data were filtered according to the maximum number of missing value allowed for each miRNA (15\%). Unsupervised computational analysis was performed using the dist function from $\mathrm{R}$ and the euclidian method as distance measure. Hierarchical clustering was obtained with the hclust function from R, using the distance matrix previously computed and the ward method. To assess differentially expressed miRNA using LIMMA package, we estimated fold changes and standard errors between the two groups by fitting a linear model for each miRNA with the lmFit function, and then applied an empirical Bayes smoothing to 
the standard errors to the linear model. A table of the top-ranked genes was extracted from the linear model fit using the topTable function and results were saved as in a Volcano plot and a table file format.

Validation of RNA expression measurements. Validation of miRNA expression was performed by reverse transcription and real-time quantitative PCR (RT-qPCR) using Taqman microRNA Reverse Transcription Kit and detection protocol in a 7500 thermocycler (Applied Biosystems, Thermo Fisher Scientific). Validation of messenger RNA (mRNA) expression was done by reverse transcription using random hexamers and Super Script II reverse transcriptase (Thermo Fisher Scientific). RT-qPCR was performed using SYBR-Green (Applied Biosystems, Thermo Fisher Scientific). Briefly, 100-200 ng of total complementary DNA (cDNA), 50 $\mathrm{nM}$ (each) primers, and $1 \times$ SYBR-Green mixture were used in a total volume of 20 $\mu \mathrm{L}$. Murine Tet3 (\#Mm01184936_g1) and Gapdh (\#Mm99999915) Taqman probes were from Thermo Fisher. The level of expression of human miRNA (miR-150 \#000473, miR-451 \#478107, miR-494 \#478944) was expressed relative to sno202 \#001232, sno234 \#001234, RNU-44 \#001094, RNU-6B \#001093, RNU-48 \#001006, or B2M \#4326319E controls (all from Applied Biosystems, Thermo Fisher Scientific), or PPIA, HPRT, or RPL32 controls. Primer sequences: hPPIA F, 5'-GT CAACCCCACCGTGTTCTT- $3^{\prime}$ and R, 5'-CTGCTGTCTTTGGGACCTTGT-3', probe: AGCTCAAAGGAGACGCGGCCCA; hHPRT R, 5'-GGCAGTATAATCC AAAGATGGTCAA- $3^{\prime}$ and F, 5' ${ }^{\prime}$-TCAAATCCAACAAAGTCTGGCTTATAT-3', probe: 5'-CTTGCTGGTGAAAAGGACCCCACGA-3'; mB2M F, 5'-TATATCCT GGCTCACACTGAATTC-3' and R, 5'-TCGATCCCAGTAGACGGTCTT-3', probe: CCCACTGAGACTGATACATACGCCTGCA. FCGRT and TET3 expression was normalized to B2M, HPRT, RPL32, and GUS for SYBR-Green experiments. SYBR primers: hB2M F, 5'-ACACAACTGTGTTCACTAGC-3' and R, 5'CAACTTCATCCACGTTCACC-3' hHPRT F, 5'-GGACAGGACTGAACGTCTT GC-3' and R, 5'-CTTGAGCACACAGAGGGCTACA-3'; hRPL32 F, 5'-TGTCCT GAATGTGGTCACCTGA-3' and R, 5' -CTGCAGTCTCCTTGCACACCT- $3^{\prime}$; GUS F, 5'- GAAAATATGTGGTTGGAGAGCTCATT- $3^{\prime}$ and R, 5'-CCGAGTGA AGATCCCCTTTTTA-3'; hFCGRT F, 5'-GGAGCTTGGGTCTGGGAAA-3' and R, 5'-GCTTCTCCTTGATCCTCAGATCTG-3'; hTET3 F, 5'-GCGCGGCATGGT ATGAA- $3^{\prime}$ and R, $5^{\prime}$-ACTCGAGGTAGTCAGGGCATTCT- $3^{\prime}$. They were expressed as the $2-\wedge \wedge \mathrm{Ct}$.

Detection of gene mutations in CMML cells. We used Ion AmpliSeq ${ }^{\text {Tw }}$ Custom Panel Primer Pools for myeloid genes (10 ng of genomic DNA (gDNA) per primer pool) to perform multiplex PCR with slight modifications in the generation of libraries by adding indexed paired-end adaptors (NEXTflex, Scientific) before paired-end sequencing $(2 \times 250 \mathrm{bp}$ reads) using an Illumina MiSeq flow cell and the onboard cluster method (Illumina). The reads obtained were mapped to hg19 reference genome using bwa (v0.7.10). Varscan (v2.3.7) has been used to call the mutations with a frequency $>5 \%$ in each sample. The mutations were annotated with Annovar.

Mice analysis and bone marrow transplantation. White blood cell count was determined using an automated counter (MS9, Schloessing Melet, France) on blood samples collected from the retro-orbital plexus in citrated tubes. Bone marrow cells were collected by flushing femurs and tibias. Spleens were excised, weighted and pushed through a $70-\mu \mathrm{m}$ strainer. Bone marrow transplantation was performed by delivering retro-orbitaly $\sim 5 \times 10^{6}$ cells from wild-type (CD45.1) or miR-150-1(CD45.2) animals to lethally irradiated ( $9.5 \mathrm{~Gy}$ ) recipient mice (wild-type or miR$150^{-I-}$ ). For competitive transplantation experiments, lethally irradiated CD45.1 wild-type recipient mice were injected with bone marrow cells from CD45.1 wildtype donor mice mixed with cells from CD45.2 miR-150 $1-1-$ animals. Blood was analyzed at 8,12 , and 40 weeks post transplantation. MiR-150 in vivo rescue of miR-150 expression was performed by sorting $\mathrm{Lin}^{-}$bone marrow cells from miR$150^{-I-}$ mice with immunomagnetic beads (Biotin Mouse Lineage Depletion Cocktail, BD Biosciences, San Diego, CA, USA), culturing these cells for 24 to $48 \mathrm{~h}$ at $1 \times 10^{6} \mathrm{cells} / \mathrm{ml}$ in Stem Span SFEM (StemCell ref.: $5.09600 \%$ FCS, $1 \%$ penicillin/ streptomycin/L-glutamine, and $100 \mathrm{ng} / \mathrm{mL}$ SCF, $100 \mathrm{ng} / \mathrm{ml} \mathrm{TPO}, 50 \mathrm{ng} / \mathrm{ml}$, IL-6, and $10 \mathrm{ng} / \mathrm{ml} \mathrm{IL-3}$. These cells at a density of 1.5 to 2 million cells $/ \mathrm{ml}$ were subsequently transduced with the Megix-mmu-Mir150 at an MOI of 4-10. Forty-eight after transduction, 100,000 to $350,000 \mathrm{Lin}^{-}$cells (of which about $25 \%$ of $\mathrm{GFP}^{+}$ cells) were injected retro-orbitally to lethally irradiated wild-type recipients.

Monocytes adoptive transfer. Bone marrow Ly6 $6 \mathrm{C}^{\text {high }}$ monocytes were sorted from wild-type CD45.1 $1^{+}$and $m i R-150^{-1-} \mathrm{CD} 45.2^{+}$mice and mixed in a 1:1 ratio. For each wild-type $\mathrm{GFP}^{+}$mouse recipient, $4.5 \times 10^{6}$ cells in $200 \mu \mathrm{l}$ sterile phosphate-buffered saline (PBS) were injected intravenously. Recipients were sacrificed $40 \mathrm{~h}$ after injection and the transferred $\mathrm{GFP}^{-}$monocytes $\left(\mathrm{CD}^{-}, \mathrm{B}^{-} 20^{-}\right.$, $\left.\mathrm{NK}_{1.1}{ }^{-}, \mathrm{Ly}_{6 \mathrm{G}}{ }^{-}, \mathrm{CD}_{11} \mathrm{~b}^{+}, \mathrm{CD} 115^{+}\right)$were analyzed by flow cytometry.

Flow cytometry analysis. Mouse bone marrow cells were kept on ice during staining and analysis. Blood leukocytes were sorted on dextran, washed in PBS with $2 \mathrm{mM}$ EDTA and kept on ice. Analysis of progenitors was made by staining $3 \times 10^{6}$ bone marrow cells with anti-B220, Ter119, CD11b, GR1, CD3, IL-7R PE and CD117, Sca-1, CD34, and CD16/32 antibodies. Mature cell populations were identified by collecting two to three million of cells from blood or bone marrow samples in PBS $+2 \mathrm{mM}$ EDTA $+0.5 \%$ bovine serum albumin. After blockade of $\mathrm{Fc} \gamma$ receptor for $15 \mathrm{~min}$, cells were incubated with antibodies for $30 \mathrm{~min}$ at $4{ }^{\circ} \mathrm{C}$. For May-Grünwald-Giemsa staining, monocyte subsets were sorted according to gating strategies in Supplementary Figure 3, centrifuged on microscope slides, dried for $1 \mathrm{~h}$ at room temperature, and stained with May-Grünwald-Giemsa stain, or used to collect total RNA. To detect apoptosis, cells were additionally stained with Annexin V-FITC antibody (BD Biosciences) and 7-amino-actinomycin D (Invitrogen) according to the manufacturer's protocols. Cell fluorescence was determined using a Fortessa (BD Biosciences) and analyzed with the Kaluza Software (Beckman Coulter). Fluorescence intensity was expressed as staining index, using the median and slope distributions of labeled and background cells. The absolute number of cells was calculated by multiplying the percentage of cells in a subset by the leukocyte cell count before staining. For cell analysis after transfer, SYTOX ${ }^{m}$ Blue Dead Cell Stain was used to exclude dead cells (Thermo Fisher). Human mononuclear cells were isolated from peripheral blood samples using density centrifugation Pancoll (Pan-Biotech, Dutscher, Brumath, France). Monocyte subsets repartition was quantified using CD45, CD24, CD56, CD14, and CD16 antibodies (Supplementary Table 5) as described ${ }^{20}$. For monocyte quantification in differentiation assays, CD163, CD71, CD14, and CD16 antibodies were used (Supplementary Table 5). Fluorescence minus one samples were made for each cytometry antibody to assure the correct gating of stained cells (Supplementary Figure 6C, D).

Cell sorting. Mouse monocytes were first enriched from blood or bone marrow mononuclear cells by negative selection using biotinylated anti-Ly6G, B220, CD3, NK1.1, and Ter119 antibodies (Supplementary Table 5) and magnetic beads (Streptavidin particles plus, BD Biosciences). Monocyte subsets were then sorted using Aria III, Aria-Fusion or Influx (BD Biosciences and BD Diva Software) cell sorters using Streptavidin-PE, CD11b-Pacific blue, CD115-BV605, and Ly6C-APC antibodies (Supplementary Table 5) as $\mathrm{Lin}^{-}$(CD3, B220, Ter119, Nk1.1, and Ly6G-biotin/Streptavidin-PE ${ }^{+}$cells) $\mathrm{CD}_{11 b^{+}}, \mathrm{CD}_{115^{+}}$monocytes, and separated according to Ly6C expression (Supplementary Figure 3B). Human $\mathrm{CD}_{14}{ }^{+}$cells were sorted from mononuclear cells using CD14 microbeads and the AutoMacs system (Miltenyi Biotech). Monocytes subsets were first enriched from mononuclear cells by negative selection using $\mathrm{CD} 19^{-}, \mathrm{CD} 56^{-}, \mathrm{CD}^{-}, \mathrm{CD} 16^{-}$ microbeads and the AutoMacs system (Miltenyi Biotech), and then sorted using a cell sorter and CD45, CD14, and CD16 antibodies after exclusion of CD56 ${ }^{+}$cells (Supplementary Table 5). CD $34^{+}$cells were sorted from human cord blood samples using magnetic beads and the AutoMacs system (Miltenyi Biotech).

Plasmid constructs, viral production, and titration. Murine or human gDNA was used to amplify either the murine mir-150 sequence (mmu-miR-150) using the primers mmu-miR-150-fwd: 5'-GAC AGG AAC CCC CTC CCT CAG-3' and mmu-miR1-50-rev: 5'-AGG AAG GGA CCC AAG GCA TCC-3', or the human hsa-miR-150 sequence (hsa-miR-150) using the primers hsa-miR-150-fwd: 5'-GTA ACG CGT CTG GAC CTG GGT ATA AGG CA-3' and hsa-miR-150-rev: 5'-GTA GCT AGC GCA GCA GAG ATG GGA GTA CA- $3^{\prime}$ containing a $M l u \mathrm{I}$ and NheI restriction site, respectively. PCR fragments were cloned in TOPO2.1 (Thermo Fisher ref.: K450001) according to the manufacturer's instructions, and then transferred to the Megix retroviral backbone after an EcoRI digestion (mmir150) or to the pRRL lentiviral backbone (sinpRRL-PGK-GFP) after a MluI/NheI double digestion (hsa-miR-150). Human miRZip-150 anti-miR-150 microRNA construct (MZIP150-PA-1, Ozyme, France) was used to amplify the H1 promoter and the anti-miR-150 using the forward primer, $5^{\prime}$-CGCCTCGAGATATTTGCATGTCGC TATGTGTTCTGGG- $3^{\prime}$ and the reverse primer, ${ }^{\prime}$-CGCCTCGAGGAATTCAAA AATCTCCCAACCCTTGTAC-3', both with a XhoI restriction site. The PCR fragment was cloned into the pRRL lentiviral backbone after XhoI digestion and dephosphorylation. CRISPR lentiviral vector constructs: the backbone vector was constructed from the LentiCRISPR-V2puro (Addgene, \#52961) to co-express Streptococcus pyogenes Cas9 linked to the GFP sequence by a P2A peptide driven by elongation factor-1 short. Two gRNAs (289 and 471) were selected (CRISPOR tools) to specifically delete the $\mathrm{R} 3$ region. In order to clone the guide sequence into the single-guide RNA scaffold, we annealed either the primer gRNA289-fwd, 5'CAC CGT TTA ATA TCC TTC TGC GGG C-3' with gRNA289-rev, 5'-AAA CGC CCG CAG AAG GAT ATT AAA C- $3^{\prime}$ for the gRNA289 or the primer gRNA471fwd, 5'-CAC CGC GAG TGA GGC AGC ATT GTA T- ${ }^{\prime}$ ' with gRNA471-rev, 5' $^{\prime}$ AAA CAT ACA ATG CTG CCT CAC TCG C-3' for the gRNA471. This annealing resulted in a four paired sticky end compatible with a BsmBI digestion. Thus, the CRISPR backbone was digested by BsmBI and ligated with the annealed gRNAs. Orientations and sequences were verified by Sanger sequencing. High-titer stocks of retroviral or lentiviral vectors were produced by transient tripartite JetPrime transfection of $293 \mathrm{~T}$ cells with (i) the vector plasmid, (ii) an encapsidation plasmid, and (iii) a vesicular stomatitis virus envelope (VSV-g) plasmid according to the manufacturer's instructions (Polyplus, \#114-07). Forty-eight hours after transfection, supernatants were concentrated by ultracentrifugation. Vector titers were determined by transduction of serial dilutions of the concentrated supernatant in 293T cells and measurement of GFP expression. Plasmid constructs and U937 transduction for GFP reporter assay are described in Supplementary methods. 
ChIP-sequencing. Cells were cross-linked with addition of $1 \%$ formaldehyde to the culture medium for $10 \mathrm{~min}$ at room temperature with agitation. Fixation was stopped by the addition of $125 \mathrm{mM}$ glycine during $5 \mathrm{~min}$ at room temperature with agitation before washing samples twice in ice-cold PBS and adding sodium dodecyl sulfate lysis buffer (Millipore, $10 \mu \mathrm{l}$ per $1 \times 10^{6}$ cells) supplemented with $1 \%$ protease inhibitor cocktail (Active Motif). Samples were incubated $15 \mathrm{~min}$ on ice before storage at $-80^{\circ} \mathrm{C}$. Cross-linked DNA were sonicated using $12 \times 24 \mathrm{~mm}^{2}$ round bottom tube, $10 \mathrm{~min}$ at $40 \mathrm{~W}$ (Covaris S220, Woodingdean, UK). ChIP was carried out using ChIP-it Express Kit according to the manufacturer's instruction (Active Motif). Enriched DNA from ChIP and Input DNA fragments were endrepaired, extended with an "A" base on the 3 ' end, ligated with indexed paired-end adaptors (NEXTflex, Bioo Scientific, Proteigene, Saint Marcel, France) using the Bravo Platform (Agilent, Les Ulis, France), size selected after four cycles of PCR with AMPure XP beads (Beckman Coulter, Villepinte, France), and amplified by PCR for 10 more cycles. Fifty-cycle single-end sequencing was performed using HiSeq 2000 (Illumina) for controls and CMML CD14 ${ }^{+}$monocytes, or fifty-cycle single-end sequencing with NovaSeq 6000 (Illumina) for control monocyte subsets, in order to reach at least 20 millions reads per sample. Reads were aligned into human genome hg19 with BWA aln (v0.7.5a) and peak calling assessed using MACS 2.0 with a $q$ value cut-off of 0.05 for the histone mark H3K4me3. Annotation has been done with HOMER (v4.7.2), with a $P$ value of 0.01 . Integrative Genomics Viewer (IGV 2.1) was used for representation. Normalized bigwig files have been computed using bamcomverage from deeptools 3.1.2, with a bin size of 50 , the number of read per bin normalized using RPKM methods, reads extension set to $250 \mathrm{bp}$, and a smooth length windows of $150 \mathrm{bp}$. Reads with the same orientation and start have been considered only once. From normalized bigwig files, the distribution of signal for each mark and the corresponding heatmap have been computed using computeMatrix and plotHeatmap from deeptools 3.1.2, using TSS from Human genome annotation file (genecode.v19.annotation.gft) as the reference point

Bisulfite DNA sequencing. Two hundred nanograms of total gDNA were modified by bisulfite treatment according to the manufacturer's instructions (MethylDetector, Active Motif). Converted R3 miR-150 promoters were identified by PCR using primers MIR150convR3 F2, 5'-GGTATTTTTTTAAAAAATTTTTGTAGG TG-3' andR, 5' -CTCTATTACCAATCTAAAATACAATAATACAATC- ${ }^{\prime}$, and direct sequencing reaction was performed using standard conditions (Applied Biosystems)

Protein extraction and immunoblotting. Sorted monocytes were lysed in $2 \times$ Laemmli with $0.1 \mathrm{M}$ dithiothreitol, boiled $10 \mathrm{~min}$ at $95^{\circ} \mathrm{C}$, and sonicated to collect total proteins, which were separated on polyacrylamide gel and transferred to nitrocellulose membrane (Thermo Fisher Scientific). Membranes were blocked with $5 \%$ bovine serum albumin in PBS, with $0.1 \%$ Tween-20 (Sigma-Aldrich) for $40 \mathrm{~min}$ at room temperature, incubated overnight at $4{ }^{\circ} \mathrm{C}$ with the primary antibodies, washed in PBS-0.1\% Tween-20, incubated further with horse radish peroxidase-conjugated secondary antibody $(400 \mathrm{ng} / \mathrm{ml})$ for $1 \mathrm{~h}$ at room temperature and washed again before analysis using Immobilon Western Chemiluminescent HPR Substrate system (Millipore, Molsheim, France). The chemiluminescent emission was registered by imageQuant LAS 4000 camera (GE Healthcare Life Science, Vélizy, France). Uncropped scans of key immunoblots are shown in Supplementary Figure 11.

miR-150 pull-down. Monocytes were sorted with Monocyte Isolation Kit II (Miltenyi Biotech) before resuspending $5 \times 10^{6}$ cells in $100 \mu$ nucleofector solution with 2 pM hsa-miR-150 or miR-39p, a control mi-RNA from Caenorhabditis elegans (miRcury LNA microRNA mimic, Exiqon). Cells were electroporated in duplicate (Lonza, Amboise, France), cultured overnight at $10^{6} / \mathrm{ml}$ in RPMI-1640 Glutamax medium (Thermo Fisher Scientific) supplemented with $10 \%$ heatinactivated FBS, $1 \%$ penicillin/streptomycin, and $2 \mathrm{mM}$ L-glutamine (Thermo Fisher Scientific) for $20 \mathrm{~h}$, and lysed. MicroRNA pull-down was then performed as described $^{70}$. Briefly, monocytes were washed twice with PBS and resuspended in a lysis buffer. After $20 \mathrm{~min}$ on ice, lysates were spun at $5000 \times g$ for $5 \mathrm{~min}$ at $4{ }^{\circ} \mathrm{C}$. The supernatant was incubated with magnetic beads (Dynabeads, Life Technologies) for $4 \mathrm{~h}$ on a rotator. Beads were washed five times in the lysis buffer and resuspended in $100 \mu \mathrm{l}$ of lysis buffer. RNAs were isolated with $500 \mu \mathrm{l}$ of Trizol LS (Life Technologies). RNA obtained were purified on Amicon ultra- 0.5 centrifugal filter devices (Millipore). Four independent experiments were made on distinct buffy coats. Two hundred picograms of concentrated RNA were first-strand synthesized and tailed using SMARTScribe reverse transcriptase (SMARTer Universal Low Input RNA, Clontech). The cDNA strand was then template switched and extended by SMARTScribe reverse transcriptase. Double-stranded cDNA was amplified by 25 cycles of PCR using Advantage 2 PCR Kit (Clontech), purified by AMPure beads (were end-repaired, extended with an " $A$ " base on the 3 ' end, ligated with indexed paired-end adaptors (NEXTflex, Bioo Scientific) using the Bravo Platform (Agilent) and amplified by PCR for 10 cycles. Indexed libraries were sequenced in an Illumina MiSeq flow cell using the onboard cluster method, as paired-end sequencing $(2 \times 250 \mathrm{bp}$ reads) (Illumina, San Diego, CA, USA). The reads were adapter and quality trimmed with Trimmomatic (v0.32). Cleaned reads were mapped to hg19 reference genome with Tophat2 (v2.0.14)/Bowtie2 (v2.1.0) with the following parameters: --read-realign-edit-dist 0 --library-type frunstranded. The regions covered were identified with Bedtools (v2.17.0) and annotated with Homer (v4.7.2).

RNA-sequencing. RNA integrity (RNA integrity score $\geq 7.0$ ) was checked on the Agilent 2100 Bioanalyzer (Agilent) and quantity was determined using Qubit (Invitrogen). SureSelect Automated Strand Specific RNA Library Preparation Kit was used according to the manufacturer's instructions with the Bravo Platform. Briefly, $100 \mathrm{ng}$ of total RNA sample was used for poly-A mRNA selection using oligo $(\mathrm{dT})$ beads and subjected to thermal mRNA fragmentation. The fragmented mRNA samples were subjected to cDNA synthesis and were further converted into double-stranded DNA using the reagents supplied in the kit, and the resulting double-stranded DNA was used for library preparation. The final libraries were sequenced on an Hiseq 2000 for human samples and on NovaSeq 6000 for mice samples (Illumina) in paired-end $100 \mathrm{bp}$ mode in order to reach at least 30 millions reads per sample at Gustave Roussy.

For human samples analysis, raw reads were mapped to hg19 genome with Tophat2 (v2.0.14)/Bowtie2 (v2.1.0) with the following parameters: --read-realignedit-dist 0 --library-type fr-firststrand --mate-inner-dist 50. Expression analysis was performed by counting the number of reads per gene using HTSeq (0.5.4p5) with the following parameters: -m union -s reverse -a 10 and GENECODE gene annotation (v24lift37). DESeq2 (v1.10.1) package was used for differential gene expression analysis.

For mouse sample analysis, Fastq files quality have been analyzed with FastQC (v0.11.7) and aggregated with MultiQC (v1.5). The quantification was performed on Gencode mouse M18 (GRCm38p6) transcriptome and comprehensive gene annotation, with Salmon (v0.10.2). The index was build with the default k-mer length of 31 , with the genecode flag on, the perfect hash option, and all 1569 sequence duplicates within the genome were kept. The quantification was done with the default expected maximization algorithm, verified through 100 boostrap rounds, sequence-specific bias correction, fragment GC-bias correction, and automatic library detection parameter. Clustered heatmaps were performs from normalized counts with pheatmap, an R package, using Pearson's coefficient as distance metric for rows and column and the Ward.D2 method for the clustering. Volcano plot were built using ggplot package. The differential analysis was performed with Sleuth (v0.29.0), on data converted by wasabi (v0.2)

Statistical analysis. Student's $t$ test were performed using the Prism software.

\section{Data availability}

Datasets are available in the ArrayExpress database at EMBL-EBI (www.ebi.ac.uk/ arrayexpress). H3K4me3 CHIP-Seq comparing CMML and healthy donor monocytes profiles are referenced as E-MTAB-6689 and those comparing human classical, intermediate, or nonclassical monocytes profiles are referenced as EMTAB-7290. RNA-sequencing comparing CMML and healthy donor monocytes expression profiles are referenced as E-MTAB-6712 and those RNA-sequencing comparing classical or nonclassical monocytes expression profiles between wildtype and $\mathrm{MiR}-150^{-1-}$ or wild-type and $\mathrm{Tet} 3^{-1-}$ mice are referenced as E-MTAB7291. GRO-cap analyses obtained in a human myeloid (K562) and a human lymphoid (GM12878) cell line (Supplementary Figure 7A) were collected from www.ncbi.nlm.nih.gov/geo/ (GSE60456 accession). ChIP-seq data for H3K27me3, $\mathrm{H} 3 \mathrm{~K} 4 \mathrm{me} 1, \mathrm{H} 3 \mathrm{~K} 4 \mathrm{me} 3$, and $\mathrm{H} 3 \mathrm{~K} 27 \mathrm{ac}$ obtained in human $\mathrm{CD}^{+}, \mathrm{CD}^{+} 9^{+}, \mathrm{CD}_{5}{ }^{+}$, and $\mathrm{CD} 14^{+}$cells (Fig. 5C) were collected from www.roadmapepigenomics.org/. GSM1027287_UW.CD19_Primary_Cells.H3K27ac.RO_01701.Histone.DS21712.wig: GSM1027288_UW.CD56_Primary_Cells.H3K27ac.RO_01701.Histone.DS21716.wig: GSM1027290_UW.CD19_Primary_Cells.H3K27me3.RO_01679.Histone.DS22585.wig: GSM1027291_UW.CD56_Primary_Cells.H3K27me3.RO_01679.Histone.DS22594.wig: GSM1027296 UW.CD19_Primary_Cells.H3K4me1.RO_01679.Histone.DS22584.wig: GSM1027297_UW.CD56_Primary_Cells.H3K4me1.RO_01679.Histone.DS22593.wig: GSM1027300_UW.CD19_Primary_Cells.H3K4me3.RO_01701.Histone.DS21711.wig: GSM1027301_UW.CD56_Primary_Cells.H3K4me3.RO_01701.Histone.DS21715.wig: GSM1027304_UW.CD19_Primary_Cells.Input.RO_01701.Histone.DS21628.wig: GSM1027305_UW.CD56_Primary_Cells.Input.RO_01701.Histone.DS21629.wig: GSM1058764_UW.CD3_Primary_Cells.H3K27ac.RO_01701.Histone.DS21704.wig: GSM1058778_UW.CD3_Primary_Cells.H3K4me1.RO_01679.Histone.DS22561.wig: GSM1058782_UW.CD3_Primary_Cells.H3K4me3.RO_01701.Histone.DS21703.wig : GSM1058789_UW.CD3_Primary_Cells.Input.RO_01701.Histone.DS21626.wig: GSM1102782_UW.CD14_Primary_Cells.H3K27ac.RO_01721.Histone.DS22926.wig: GSM1102785_UW.CD14_Primary_Cells.H3K27me3.RO_01721.Histone.DS22405.wig: GSM1102787_UW.CD3_Primary_Cells.H3K27me3.RO_01679.Histone.DS22927.wig: GSM1102793_UW.CD14_Primary_Cells.H3K4me1.RO_01721.Histone.DS22403.wig: GSM1102797_UW.CD14_Primary_Cells.H3K4me3.RO_01701.Histone.DS21707.wig: GSM1102807_UW.CD14_Primary_Cells.Input.RO_01701.Histone.DS21627.wig

Received: 6 February 2018 Accepted: 19 November 2018 Published online: 21 December 2018 


\section{References}

1. Geissmann, F., Jung, S. \& Littman, D. R. Blood monocytes consist of two principal subsets with distinct migratory properties. Immunity 19, 71-82 (2003).

2. Carlin, L. M. et al. Nr4a1-dependent Ly6C(low) monocytes monitor endothelial cells and orchestrate their disposal. Cell 153, 362-375 (2013).

3. Shi, C. \& Pamer, E. G. Monocyte recruitment during infection and inflammation. Nat. Rev. Immunol. 11, 762-774 (2011).

4. Ziegler-Heitbrock, L. et al. Nomenclature of monocytes and dendritic cells in blood. Blood 116, e74-e80 (2010).

5. Cros, J. et al. Human CD14dim monocytes patrol and sense nucleic acids and viruses via TLR7 and TLR8 receptors. Immunity 33, 375-386 (2010).

6. Zhu, Y. P., Thomas, G. D. \& Hedrick, C. C. 2014 Jeffrey M. Hoeg Award Lecture: transcriptional control of monocyte development. Arterioscler. Thromb. Vasc. Biol. 36, 1722-1733 (2016).

7. Drissen, R. et al. Distinct myeloid progenitor-differentiation pathways identified through single-cell RNA sequencing. Nat. Immunol. 17, 666 (2016).

8. Notta, F. et al. Distinct routes of lineage development reshape the human blood hierarchy across ontogeny. Science 351, aab2116 (2016).

9. Olsson, A. et al. Single-cell analysis of mixed-lineage states leading to a binary cell fate choice. Nature 537, 698-702 (2016).

10. Kawamura, S. et al. Identification of a human clonogenic progenitor with strict monocyte differentiation potential: a counterpart of mouse cMoPs. Immunity 46, 835-848 (2017). e4

11. Yáñez, A. et al. Granulocyte-monocyte progenitors and monocyte-dendritic cell progenitors independently produce functionally distinct monocytes. Immunity 47, 890-902 (2017). e4.

12. Hanna, R. N. et al. The transcription factor NR4A1 (Nur77) controls bone marrow differentiation and the survival of Ly6 $\mathrm{C}^{-}$monocytes. Nat. Immunol. 12, 778-785 (2011).

13. Thomas, G. D. et al. Deleting an Nr4a1 super-enhancer subdomain ablates Ly6Clow monocytes while preserving macrophage gene function. Immunity 45, 975-987 (2016)

14. Wong, K. L. et al. Gene expression profiling reveals the defining features of the classical, intermediate, and nonclassical human monocyte subsets. Blood 118, e16-e31 (2011)

15. Zawada, A. M. et al. SuperSAGE evidence for $\mathrm{CD} 14^{++} \mathrm{CD} 16^{+}$monocytes as a third monocyte subset. Blood 118, e50-e61 (2011).

16. Patel, A. A. et al. The fate and lifespan of human monocyte subsets in steady state and systemic inflammation. J. Exp. Med. https://doi.org/10.1084/ jem.20170355 (2017)

17. Hofer, T. P. et al. Slan-defined subsets of CD16-positive monocytes: impact of granulomatous inflammation and M-CSF receptor mutation. Blood 126, 2601-2610 (2015).

18. Stansfield, B. K. \& Ingram, D. A. Clinical significance of monocyte heterogeneity. Clin. Transl. Med. 4, https://doi.org/10.1186/s40169-014-0040-3 (2015).

19. Wong, K. L. et al. The three human monocyte subsets: implications for health and disease. Immunol. Res. 53, 41-57 (2012).

20. Selimoglu-Buet, D. et al. Characteristic repartition of monocyte subsets as a diagnostic signature of chronic myelomonocytic leukemia. Blood $\mathbf{1 2 5}$ 3618-3626 (2015).

21. Deininger, M. W. N., Tyner, J. W. \& Solary, E. Turning the tide in myelodysplastic/myeloproliferative neoplasms. Nat. Rev. Cancer 17, 425-440 (2017).

22. Itzykson, R. et al. Clonal architecture of chronic myelomonocytic leukemias. Blood 121, 2186-2198 (2013).

23. Mason, C. et al. Age-related mutations and chronic myelomonocytic leukemia. Leukemia 30, 906-913 (2016).

24. Merlevede, J. et al. Mutation allele burden remains unchanged in chronic myelomonocytic leukaemia responding to hypomethylating agents. Nat Commun. 7, 10767 (2016)

25. Selimoglu-Buet, D. et al. Accumulation of classical monocytes defines a subgroup of MDS that frequently evolve into CMML. Blood https://doi.org/ 10.1182/blood-2017-04-779579 (2017).

26. Talati, C. et al. Monocyte subset analysis accurately distinguishes CMML from MDS and is associated with a favorable MDS prognosis. Blood 129, 1881-1883 (2017).

27. Aucagne, R. et al. Transcription intermediary factor $1 \gamma$ is a tumor suppressor in mouse and human chronic myelomonocytic leukemia. J. Clin. Invest. 121, 2361-2370 (2011).

28. Solary, E. \& Itzykson, R. How I treat chronic myelomonocytic leukemia. Blood 130, 126-136 (2017).

29. Arber, D. A. et al. The 2016 revision to the World Health Organization classification of myeloid neoplasms and acute leukemia. Blood 127, 2391-2405 (2016)
30. Xiao, C. et al. MiR-150 controls B cell differentiation by targeting the transcription factor c-Myb. Cell 131, 146-159 (2007).

31. Smith, N. L., Wissink, E. M., Grimson, A. \& Rudd, B. D. miR-150 regulates differentiation and cytolytic effector function in $\mathrm{CD}^{+} \mathrm{T}$ cells. Sci. Rep. 5, 16399 (2015).

32. Bezman, N. A., Chakraborty, T., Bender, T. \& Lanier, L. L. miR-150 regulates the development of NK and iNKT cells. J. Exp. Med. 208, 2717-2731 (2011)

33. Zheng, Q., Zhou, L. \& Mi, Q.-S. MicroRNA miR-150 is involved in Val4 invariant NKT cell development and function. I. Immunol. (Baltimore, $M d$ 1950) 188, 2118-2126 (2012)

34. Lu, J. et al. MicroRNA-mediated control of cell fate in megakaryocyte-erythrocyte progenitors. Dev. Cell 14, 843-853 (2008).

35. Adams, B. D. et al. An in vivo functional screen uncovers miR-150-mediated regulation of hematopoietic injury response. Cell Rep. 2, 1048-1060 (2012).

36. Tamura, A. et al. C/EBP $\beta$ is required for survival of Ly6C $\mathrm{C}^{-}$monocytes. Blood 130, 1809-1818 (2017).

37. Mildner, A. et al. Genomic characterization of murine monocytes reveals $\mathrm{C} /$ $\mathrm{EBP} \beta$ transcription factor dependence of Ly6C ${ }^{-}$cells. Immunity 46, 849-862 (2017). e7.

38. Schaefer, B. C., Schaefer, M. L., Kappler, J. W., Marrack, P. \& Kedl, R. M. Observation of antigen-dependent $\mathrm{CD} 8^{+} \mathrm{T}$-cell/ dendritic cell interactions in vivo. Cell. Immunol. 214, 110-122 (2001).

39. Mraz, M. et al. miR-150 influences B-cell receptor signaling in chronic lymphocytic leukemia by regulating expression of GAB1 and FOXP1. Blood 124, 84-95 (2014)

40. Lenhard, B., Sandelin, A. \& Carninci, P. Metazoan promoters: emerging characteristics and insights into transcriptional regulation. Nat. Rev. Genet. 13 233-245 (2012).

41. Andersson, R. et al. An atlas of active enhancers across human cell types and tissues. Nature 507, 455-461 (2014).

42. Schmidl, C. et al. Transcription and enhancer profiling in human monocyte subsets. Blood 123, e90-e99 (2014).

43. Fang, Z. H. et al. miR-150 exerts antileukemia activity in vitro and in vivo through regulating genes in multiple pathways. Cell Death Dis. 7, e2371 (2016).

44. He, Y., Jiang, X. \& Chen, J. The role of miR-150 in normal and malignant hematopoiesis. Oncogene 33, 3887-3893 (2014).

45. Hoareau-Aveilla, C. et al. Reversal of microRNA-150 silencing disadvantages crizotinib-resistant NPM-ALK(+) cell growth. J. Clin. Invest. 125, 3505-3518 (2015).

46. Machová Poláková, K. et al. Expression patterns of microRNAs associated with CML phases and their disease related targets. Mol. Cancer 10, 41 (2011).

47. Jiang, X. et al. Blockade of miR-150 maturation by MLL-fusion/MYC/LIN-28 is required for MLL-associated leukemia. Cancer Cell 22, 524-535 (2012).

48. Zhou, B., Wang, S., Mayr, C., Bartel, D. P. \& Lodish, H. F. miR-150, a microRNA expressed in mature B and T cells, blocks early B cell development when expressed prematurely. Proc. Natl Acad. Sci. USA 104, 7080-7085 (2007).

49. Etzrodt, M. et al. Regulation of monocyte functional heterogeneity by miR146a and Relb. Cell Rep. 1, 317-324 (2012).

50. Merkerova, M., Belickova, M. \& Bruchova, H. Differential expression of microRNAs in hematopoietic cell lineages. Eur. J. Haematol. 81, 304-310 (2008).

51. Zawada, A. M. et al. MicroRNA profiling of human intermediate monocytes Immunobiology 222, 587-596 (2017).

52. Villani, A. -C. et al. Single-cell RNA-seq reveals new types of human blood dendritic cells, monocytes, and progenitors. Science 356, pii: eaah4573 (2017).

53. Ginhoux, F. \& Jung, S. Monocytes and macrophages: developmental pathways and tissue homeostasis. Nat. Rev. Immunol. 14, 392-404 (2014).

54. Sunderkötter, C. et al. Subpopulations of mouse blood monocytes differ in maturation stage and inflammatory response. J. Immunol. (Baltim. Md, 1950) 172, 4410-4417 (2004).

55. Yona, S. et al. Fate mapping reveals origins and dynamics of monocytes and tissue macrophages under homeostasis. Immunity 38, 79-91 (2013).

56. Yrlid, U., Jenkins, C. D. \& MacPherson, G. G. Relationships between distinct blood monocyte subsets and migrating intestinal lymph dendritic cells in vivo under steady-state conditions. J. Immunol. (Baltim., Md 1950) 176, 4155-4162 (2006).

57. Korkosz, M., Bukowska-Strakova, K., Sadis, S., Grodzicki, T. \& Siedlar, M. Monoclonal antibodies against macrophage colony-stimulating factor diminish the number of circulating intermediate and nonclassical $(\mathrm{CD} 14(++)$ CD16(+)/CD14 $(+)$ CD16(++)) monocytes in rheumatoid arthritis patient. Blood 119, 5329-5330 (2012).

58. MacDonald, K. P. A. et al. An antibody against the colony-stimulating factor 1 receptor depletes the resident subset of monocytes and tissue- and tumorassociated macrophages but does not inhibit inflammation. Blood 116, 3955-3963 (2010) 
59. Rademakers, R. et al. Mutations in the colony stimulating factor 1 receptor (CSF1R) gene cause hereditary diffuse leukoencephalopathy with spheroids. Nat. Genet. 44, 200-205 (2011).

60. Landsman, L. et al. CX3CR1 is required for monocyte homeostasis and atherogenesis by promoting cell survival. Blood 113, 963-972 (2009).

61. Debien, E. et al. S1PR5 is pivotal for the homeostasis of patrolling monocytes. Eur. J. Immunol. 43, 1667-1675 (2013).

62. Ko, M. et al. TET proteins and 5-methylcytosine oxidation in hematological cancers. Immunol. Rev. 263, 6-21 (2015)

63. Abdel-Wahab, O. et al. Genetic characterization of TET1, TET2, and TET3 alterations in myeloid malignancies. Blood 114, 144-147 (2009).

64. Song, S. J. et al. MicroRNA-antagonism regulates breast cancer stemness and metastasis via TET-family-dependent chromatin remodeling. Cell 154, 311-324 (2013).

65. Lv, X., Jiang, H., Liu, Y., Lei, X. \& Jiao, J. MicroRNA-15b promotes neurogenesis and inhibits neural progenitor proliferation by directly repressing TET3 during early neocortical development. EMBO Rep. 15, 1305-1314 (2014).

66. van Furth, R. \& Cohn, Z. A. The origin and kinetics of mononuclear phagocytes. J. Exp. Med. 128, 415-435 (1968).

67. Gamrekelashvili, J. et al. Regulation of monocyte cell fate by blood vessels mediated by Notch signalling. Nat. Commun. 7, 12597 (2016).

68. Braun, T. et al. Molecular predictors of response to decitabine in advanced chronic myelomonocytic leukemia: a phase 2 trial. Blood 118, 3824-3831 (2011).

69. Quivoron, C. et al. TET2 inactivation results in pleiotropic hematopoietic abnormalities in mouse and is a recurrent event during human lymphomagenesis. Cancer Cell. 20, 25-38 (2011).

70. Tan, S. M. \& Lieberman, J. Capture and identification of miRNA targets by biotin pulldown and RNA-seq. Methods Mol. Biol. (Clifton, NJ) 1358, 211-228 (2016).

\section{Acknowledgements}

Our research was supported by the Ligue Nationale Contre le Cancer (équipes labellisées LIGUE 2017: E.S. and O.A.B.) and grants from Institut National du Cancer (INCa) to E.S. (TRANSLA13-157; PRT-K16-057; PRT-K16-067) and to O.A.B. (PLBIO16-151), Association Laurette Fugain (ALF 2017/04), Gustave Roussy taxe d'apprentissage 2009 and 2016 (to R.I. and L.B., respectively), and ANR-granted Molecular Medicine in Oncology and PACRI programs. The authors are indebted to Jeffie Lafosse for collecting clinical and biological annotations on CMML patients, and physicians and patients of the Groupe Francophone des Myélodysplasies for providing samples. We are also grateful to Enguerran Mouly and Veronique Della Valle for the establishment and maintenance of the Tet $3^{-1-}$ mice and to Françoise Porteu, Muriel Gaudry, Jean-Luc Villeval, and Fawzia Louache for scientific discussions.

\section{Author contributions}

D.S.-B. designed, performed, analyzed the experiments, and wrote the manuscript, J.R. performed cloning experiments and transduction experiments, H.G., C.L. and F.D. provided assistance for animal experiments, L.B. performed RNA-sequencing experiments on monocyte subsets, M.M. sorted monocytes and performed RNA extraction from patient samples, M.D. and G.M. performed bioinformatic analyses, M.B. prepared and performed libraries for sequencing, A.C. and C.D. performed qPCR experiments, R I., C.W., N.C. provided patient samples, O.W.-B. provided assistance for mice flow cytometry analyses, O.B. provided advices and suggestions regarding TET3 experiments, N.D. supervised molecular biology experiments, E.S. supervised the project and wrote the manuscript.

\section{Additional information}

Supplementary Information accompanies this paper at https://doi.org/10.1038/s41467018-07801-x.

Competing interests: The authors declare no competing interests.

Reprints and permission information is available online at http://npg.nature.com/ reprintsandpermissions/

Publisher's note: Springer Nature remains neutral with regard to jurisdictional claims in published maps and institutional affiliations.

\begin{abstract}
(c) (i) Open Access This article is licensed under a Creative Commons Attribution 4.0 International License, which permits use, sharing, adaptation, distribution and reproduction in any medium or format, as long as you give appropriate credit to the original author(s) and the source, provide a link to the Creative Commons license, and indicate if changes were made. The images or other third party material in this article are included in the article's Creative Commons license, unless indicated otherwise in a credit line to the material. If material is not included in the article's Creative Commons license and your intended use is not permitted by statutory regulation or exceeds the permitted use, you will need to obtain permission directly from the copyright holder. To view a copy of this license, visit http://creativecommons.org/ licenses/by/4.0\%
\end{abstract}

(C) The Author(s) 2018 